\title{
DETECTION OF NITROESTERS AND MOISTURE IN COMBUSTIBLE CARTRIDGE CASE WALL BY INDICATOR STRIPS AND INSTRUMENTS
}

\section{FINAL REPORT}

September 30, 1992

\section{Prepared for the Product Assurance and Test Directorate \\ U.S. Army Armament Research, Development and Engineering Center \\ Picatinny Arsenal \\ Dover, New Jersey $07806-5000$}

\section{By}

C.-h. Ho, J. H. Moneyhun, D. C. Agouridis,

T. M. Gayle, G. B. Hurst, and W. H. Griest

Oak Ridge National Laboratory

Oak Ridge, Tennessee 37831

This work was sponsored by the U.S. Army Armament Munitions and Chemical Command, under U.S. Department of Energy contract DE-AC05-84OR21400 with Martin Marietta Energy Systems, Inc.

\section{MASTER}




\section{EXECUTIVE SUMMARY}

Previous studies by AMCCOM, Honeywell, and ORNL have demonstrated the importance of nitroesters and moisture in the deterioration of combustible cartridge case (ccc) munitions, particularly the adhesive joint strength and the case wall hardness. Accelerated environmental exposures and modelling have predicted field lifetimes under various conditions, but considerable round-to-round variability in adhesive joint tensile strength led to large confidence intervals in predicted lifetimes. This observation suggests the need for technologies for monitoring at the individual round level to supplement lifetime predictions in the quality assurance of cec munitions.

Two approaches to individual round monitoring appear to be needed to assure munitions performance and safety. Indicator strips which could be aftixed to ccc rounds and which would change color in response to the buildup of nitroesters or moisture in the case wall would allow gun crews to rapidly determine the condition of munitions. Portable instruments which could rapidly and nondestructively measure these components in the $\mathrm{ccc}$ wall also are needed for more quantitative measurements. Such instruments could be used by the Quality Assurance Specialist - Amm:anition Surveillance in confirming the rounds identified as being defective by the indicator strips, and by ordnance personnel in the reconditioning and testing of the munitions.

In the previous phase of this project, chemistries which could detect nitroesters and moisture were screened for their suitahility in indicator strips. In this phase, those chemistries were further developed and the performance of prototype indicators was demonstrated in the laboratory. A nitroester indicator based upon a solid state version of a modified Griess reagent was found to change in color from white to red when exposed to nitroester concentrations of 0.6 to $13 \mathrm{mg} / \mathrm{g}$ in a period of several $\mathrm{d}$. The intensity of color development was proportional to the nitroester concentration of the test squares. Cupric chloride was chosen for the moisture indicator, which turns from brown to blue-green when exposed to moisture. With this indicator, the length of time required for the color change was inversely proportional to the moisture content of the test squares. Periods of 5 to 19 d were required for 6 or $8 \%$ moisture, and more than $35 \mathrm{~d}$ for a moisture concentration of $2.7 \%$. At their present stage of development, the indicator performance suggests that they would be most suitable for temporary, one-time tests of munitions rather than for permanent installation on the rounds and continuous indication. Further work on these indicators must address questions of indicator stability and lifetime, seusitivity range, potential sources of interferences, and adaptation to and calibration with actual munitions.

Two instrumental techniques were tested for their ability to determine moisture in ecc material. Electrical capacitance measurements were found to rapidly and nondestructively signify the moisture content of ece material. An inexpensive (\$325) commercially available device for determining moisture in building materials could be readily adapted to munitions. It is highly recommended that adaptation and testing of this device be continued. It appears that a working device for tield testing could be produced in the near future. Infrared spectroscopy also was briefly tested, but it did not appear at this time to be as useful or promising as the electrical capacitance technique. 
TABLE OF CONTENTS

EXECUTIVE SUMMARY $\ldots \ldots \ldots \ldots \ldots \ldots \ldots \ldots \ldots$ ii

TABLE OF CONTENTS $\ldots \ldots \ldots \ldots \ldots \ldots \ldots$ iii

LIST OF TABLES $\ldots \ldots \ldots \ldots \ldots \ldots \ldots \ldots \ldots \ldots \ldots$ iv

LIST OF FIGURES $\ldots \ldots \ldots \ldots \ldots \ldots \ldots \ldots \ldots \ldots \ldots$

INTRODUCTION $\ldots \ldots \ldots \ldots \ldots \ldots \ldots \ldots \ldots \ldots \ldots \ldots$

NITROESTER INDICATOR $\ldots \ldots \ldots \ldots \ldots \ldots \ldots \ldots \ldots \ldots \ldots$

MOISTURE INDICATOR $\ldots \ldots \ldots \ldots \ldots \ldots \ldots \ldots \ldots$

MOISTURE DETERMINATION BY DIELECTRIC MEASUREMENTS . . . . . . . . 29

PRELIMINARY EVALUATION OF INFRARED SPECTROSCOPY $\ldots \ldots \ldots \ldots$

CONCLUSIONS AND RECOMMENDATIONS $\ldots \ldots \ldots \ldots \ldots \ldots \ldots$

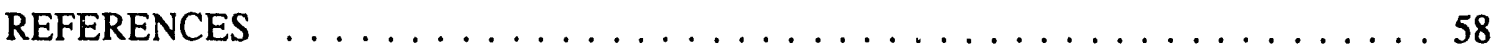

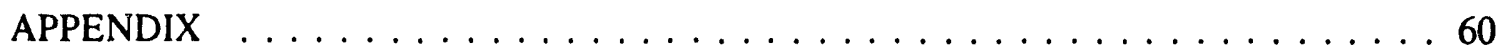




\section{LIST OF TABLES}

Table

Page

1. Relative Positions and Nitroester Concentrations

of Test Rectangles in Figure $5 \ldots \ldots \ldots \ldots \ldots \ldots \ldots$

2. Relative Positions, Reagent Configurations and Nitroester

Concentrations for Test Plate in Figures 6 and $7 \ldots \ldots \ldots 10$

3. Relative Positions of Test Strips in Figures $11-14$

and Their Mnisture Concentrations. . . . . . . . . . . . . . . . . . . . . . 19

4. Relative Positions and Moisture Concentrations of

Test Strips in Figures $15-17 \ldots \ldots \ldots \ldots \ldots \ldots$ 


\section{LIST OF FIGURES}

Figure

Page

1. Skive Joint Coupon Tensile Strength Versus

Total Nitroester Concentration $\ldots \ldots \ldots \ldots \ldots \ldots$. . . . . . . . . . 3

2. Tensile Strength Versus Total Nitroester Concentration

in Full-up M829 Rounds (4). . . . . . . . . . . . . . . . . . . . . . . 3

3. Diagram of Nitroester Indicator Structure $\ldots \ldots \ldots \ldots \ldots$

4. Mechanism of the Modified Griess Reaction Used

for the Nitroester Indicator. . . . . . . . . . . . . . . . . . . . . . 6

5. Photograph of Nitroester Indicator Strip Test Plate

After $4 \mathrm{D}$ of Exposure . . . . . . . . . . . . . . . . . . . . .

6. Photograph of Nitroester Indicator Strip

Test Plate After $1 \mathrm{D}$ of Exposure $\ldots \ldots \ldots \ldots \ldots \ldots \ldots$

7. Photograph of Nitroester Indicator Strip

Test Plate After $16 \mathrm{D}$ of Exposure ... . . . . . . . . . . . . . 12

8. Uptake of Moisture by CCC Exposed

to $95-100 \%$ RH Air . . . . . . . . . . . . . . . . . . 16

9. Moisture Uptake by CCC Placed in Water $\ldots \ldots \ldots \ldots \ldots$

10. Diagram of Moisture Indicator Design.

(Relative thicknesses are not shown to scale.) . . . . . . . . . . . . . 17

11. Photograph of Moisture Indicator

Test Plate After 4 - 5 D of Exposure . . . . . . . . . . . . . . . 20

12. Photograph of Moisture Indicator

Test Plate After $10-11 \mathrm{D}$ of Exposure $\ldots \ldots \ldots \ldots \ldots$

13. Photograph of Moisture Indicator Test Plate

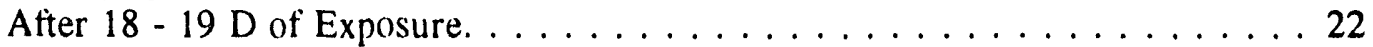

14. Photograph of Moisture Indicator Test Plate

After $84-85 \mathrm{D}$ of Exposure. . . . . . . . . . . . . . . . . . . . 23 


\section{LIST OF FIGURES \\ (CONTINUE)}

Figure

Page

15. Photograph of Moisture Indicator

Test Plate After $5 \mathrm{D}$ of Exposure. . . . . . . . . . . . . . . . . 24

16. Photograph of Moisture Indicator

Test Plate After $19 \mathrm{D}$ of Exposure. . . . . . . . . . . . . . 25

17. Photograph of Moisture Indicator

Test Plate After $35 \mathrm{D}$ of Exposure. . . . . . . . . . . . . . 26

18. Change of Capacitance (C) and Dissipation Factor (D)

of Device \#5 as a Function of Time, After Removal From

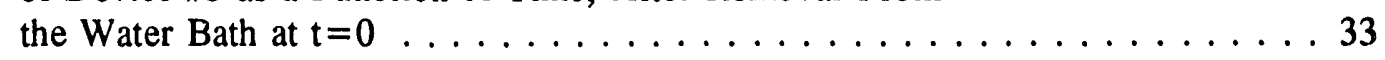

19. Capacitance vs Frequency of Device \#5. Dry and Seven Hours

After Removal from the Water Bath $\ldots \ldots \ldots \ldots$. . . . . . . . . . 34

20. Dissipation Factor vs Frequency of Device \#5,

Dry and Seven Hours After Removal from the Water Bath . . . . . . . 35

21. Capacitance (C) and Dissipation Factor (D) vs Frequency of

Device \#5. Seven Hours After Removal from the Water Bath . . . . . . . 36

22. Dielectric Constant vs Frequency. Source: Pyper $^{7} \ldots \ldots \ldots \ldots \ldots$

23. Moisture Detection Using Tramex I\#4 - Scale \#1

vs Moisture in Grams in $\mathrm{C} \# 2 \ldots \ldots \ldots \ldots \ldots$. . . . . . . . . . 40

24. Capacitance (C) and Dissipation Factor (D) of C\#1 Empty

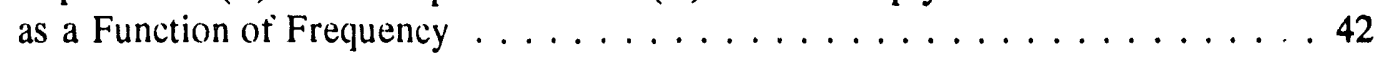

25. Capacitance (C) and Dissipation Factor (D) of C\#1, with

Wooden Cylinder Inserted, as a Function of Frequency

26. Variation of Capacitance (C) vs Time of $C \# 1$ with

Wet Cylinder at its Center . . . . . . . . . . . . . . . . . . . . 44

27. Variations of D vs Time of C\#1 with Wet Wooden Cylinder at the Center . . . . 45 


\section{LIST OF FIGURES \\ (CONTINUE)}

Figure

Page

28. Variations of the Capacitance with Frequency of C\#1

Empty and C\#1 Full of Dry Saw Dust . . . . . . . . . . . . . 47

29. Capacitance $\mathrm{C}$ vs Excess Water (dW) with the

Frequency $\mathrm{F}$ as a Parameter . . . . . . . . . . . . . . . 48

30. Magnitude of the Impedance vs Frequency with

Excess Water $(\mathrm{dW})$ as a Parameter . . . . . . . . . . . . . . . . . . . . . 49

31. Mass Changes in CCC Coupons $\ldots \ldots \ldots \ldots \ldots \ldots \ldots$

32. DRIFT Spectra of Wet (Top) and Dry (Bottom) CCC Coupon . . . . . . 55 


\section{INTRODUCTION}

Nitroester migration into the case wall from the propellant and moisture accumulation within the case have been identified (1-4) as important internal and external (respectively) factors which contribute to the physical deterioration of combustible cartridge case (ccc) munitions. The latter factor also may hinder proper ignition of the ccc and cause incomplete combustion in firing. Visual indicators sensitive to these factors and affixed to ccc rounds would allow quality assurance specialists or gun crews in the field to rapidly determine the potential reliability of individual rounds. Such visual indicator strips or bands would supplement field life predictions from accelerated lifetime testing and modelling in the assurance of munitions safety and performance.

Previous work (5) in this task identified chemistries suitable for the detection of nitroesters and moisture in the ccc wall. A version of the Griess reaction was modified for a dry colorimetric indicator which in the presence of nitroglycerin (NG) or diethyleneglycol dinitrate (DEGDN) generates a brilliant red color. Inorganic salts such as cupric chloride, which changes from brown to blue-green upon hydration, were suggested as promising visual indicators of moisture. Both chemistries are compatible with ccc.

This report describes the development and preliminary testing of prototype nitroester and moisture indicator strips, and the scoping of two instrumental techniques, infrared spectroscopy and electrical capacitance, which could lead to portable instruments for rapid and nondestructive testing of ccc in the field. 


\title{
NITROESTER INDICATOR
}

\author{
Background
}

As noted in our previous report (5), the ideal nitroester indicator strip should meet several requirements:

a. The indicator strip must be designed for placement on the surface of the ccc wall and indicate the level of total nitroesters (NG plus DEGDN) in the casing. It must not interfere with the storage, chambering, or firing of the round.

b. The color change must be readily visible.

c. The chemistry of the indicator must be compatible with the ccc, and not cause deterioration of the cec. It should not pose a hazard to gun crews.

d. The indicator strip should have a long lifetime (decades).

To estimate the nitroester concentration in ccc which is associated with significant weakening of the skive joint, correlations and predictions were made from laboratory studies of full-up rounds. In the AMCCOM study $(1,2)$ M829 rounds were exposed to different temperatures and at intervals the tensile strength of the Skive joint was determined using coupons cut from the case wall after the exposures. Nitroesters were measured in samples of the case wall taken from the bottom of the round. A linear regression was made of the tensile strength versus tcial nitroesters (NG plus DEGDN). The data with the linear regression line and the $95 \%$ confidence intervals are shown in Figure 1. The nitroester concentration corresponding to Skive joint failure $(0 \mathrm{lbs} / \mathrm{in})$ was calculated to be $122 \mathrm{mg} / \mathrm{g}$. For the more conservative Honeywell suggestion (6) of $100 \mathrm{lbs} /$ in as a minimum acceptable strength, a concentration of $76 \mathrm{mg} / \mathrm{g}$ was calculated.

In the ORNL study (4), accelerated environmental exposures of full-up M829 APFSDS rounds were followed by tensile strength tests of the whole skive joints. Nitroester analysis was performed on cec wall sections taken immediately below the skive joint for 30 rounds selected from 7 accelerated environmental exposure tests. The tensile strengths (calculated as $\mathrm{lbs} / \mathrm{in}$ from the strength of the whole joint divided by its circumference) were subjected to a linear correlation with the nitroester content. Figure 2 shows the plot with the regression line and the 95\% confidence intervals. Nitroester concentrations for two tensile strengths were then predicted from the regression. The nitroester content predicted for a tensile strength of $0 \mathrm{lbs} / \mathrm{in}$ was $147 \mathrm{mg} / \mathrm{g}$. The concentration predicted for the lowest tensile strength actually measured in this study, $15.3 \mathrm{lbs} /$ in $(227 \mathrm{lbs} / \mathrm{joint})$, was $138 \mathrm{mg} / \mathrm{g}$. This strength of $15.3 \mathrm{lbs} / \mathrm{in}$ was for a round which survived the rigors of being exposed in the test chamber, placed in its protective case and transported by truck to the production line test equipment, and 


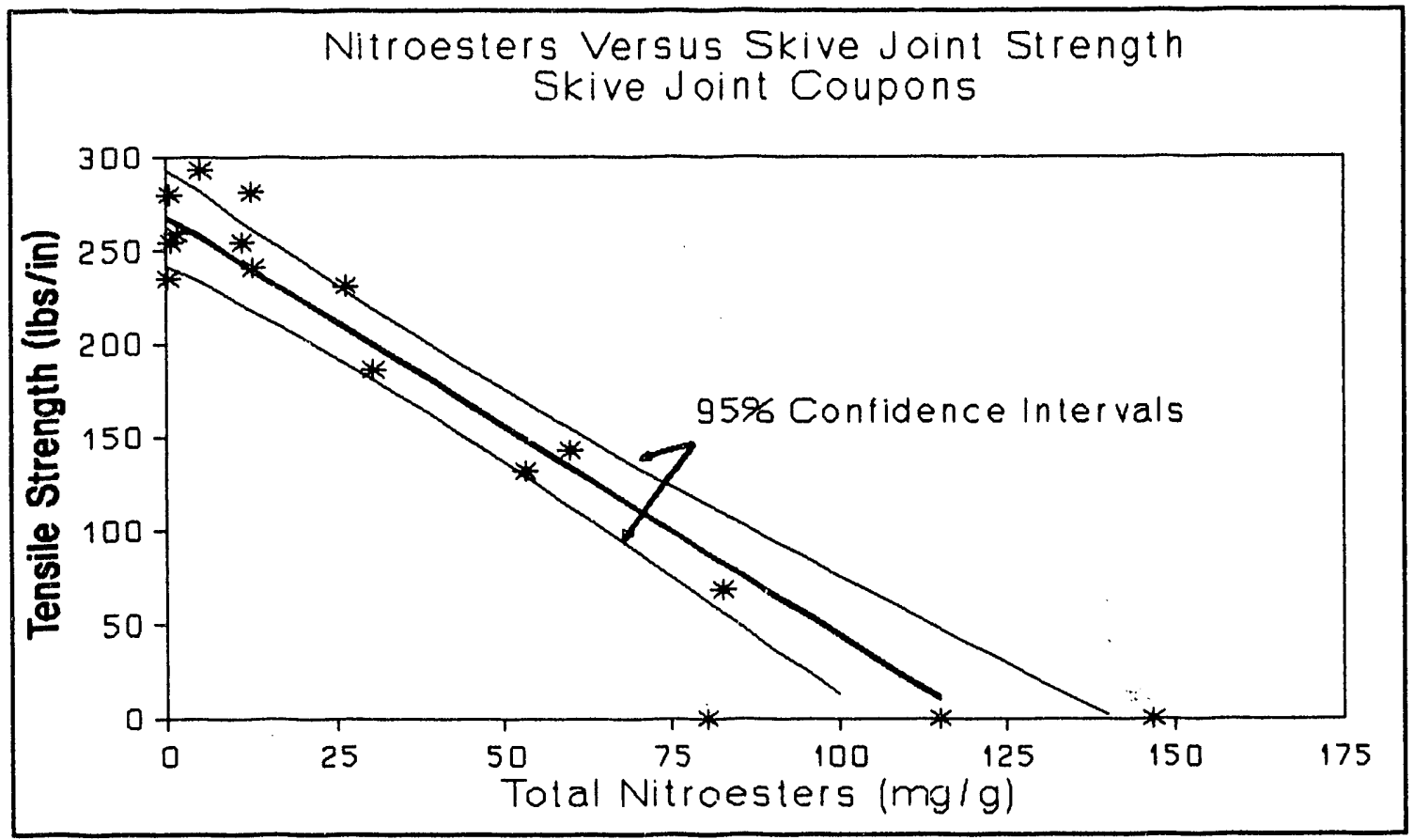

Fig. 1. Skive Joint Coupon Tensile Strength Versus Total Nitroester Concentration (2).

\section{Nitroesters Versus Skive Joint Strength \\ Full.Up M829 Rounds}

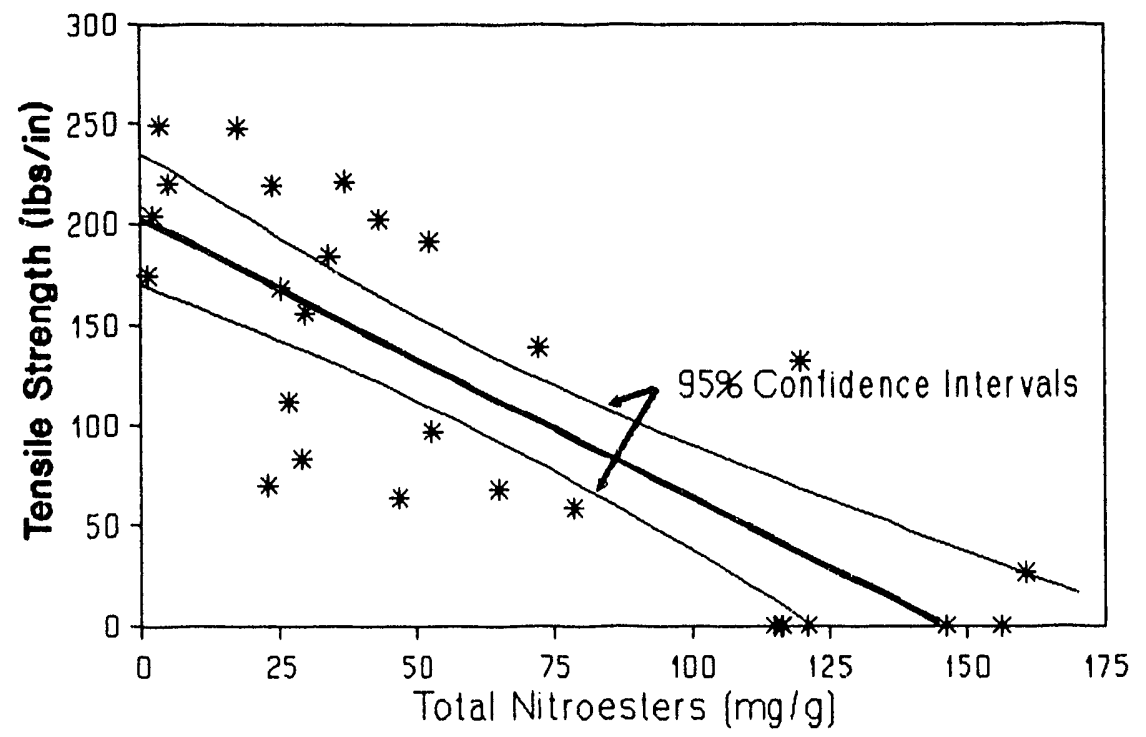

Fig. 2. Tensile Strength Versus Total Nitroester Concentration in Full-up M829 Rounds (4). 
tested for chambering with a chamber gauge before being strength-tested using a Slota apparatus. It appears to represent a low strength (but not necessarily the lowest strength) at which the Skive joint is severely weakened, but will still hold together for chambering and firing. The most conservative concentration would be for the $100 \mathrm{lbs} / \mathrm{in}$ minimum tensile strength value suggested by Honeywell (6). For this value, a nitroester content of $74 \mathrm{mg} / \mathrm{g}$ was calculated. This is in excellent agreement with the estimate from the AMCCOM data. However, the scatter in the ORNL data was considerable, because of round-to-round variability in both tensile strength and nitroester content for a given exposure condition. Therefore, a factor of 5 below the concentration equivalent to the Honeywell lower limit (ca. $15 \mathrm{mg} / \mathrm{g}$ ) was used as a conservative target for indicator sensitivity to assure that the indicator will change color before the round is severely weakened.

\section{Experimental}

\section{Test Rectangles or Squares}

White blotter paper was used to simulate the surface of a cce round. Squares of $1.9 \mathrm{~cm} \times 1.9$ $\mathrm{cm}$ dimensions and weighing $\mathrm{ca} .150 \mathrm{mg}$ each were dried in an oven at $100^{\circ} \mathrm{C}$. Known volumes of a NG standard in methanol $(1 \mathrm{mg} / \mathrm{mL})$ were pipetted onto the blotter paper squares and dried until the surface liquid disappeared. The squares were not allowed to dry completely because the limited masses of NG would not effectively diffuse into the indicator strips without traces of solvent.

\section{Indicator Strip}

The nitroester indicator strip structure is diagrammed in Figure 3. The indicator strip consisted of Nucleopore glass fiber filter with various combinations of the modified Griess reagent and powdered zinc applied to the upper or lower surfaces. The strip was sealed to the test square or rectangle using Bel Art tape. 


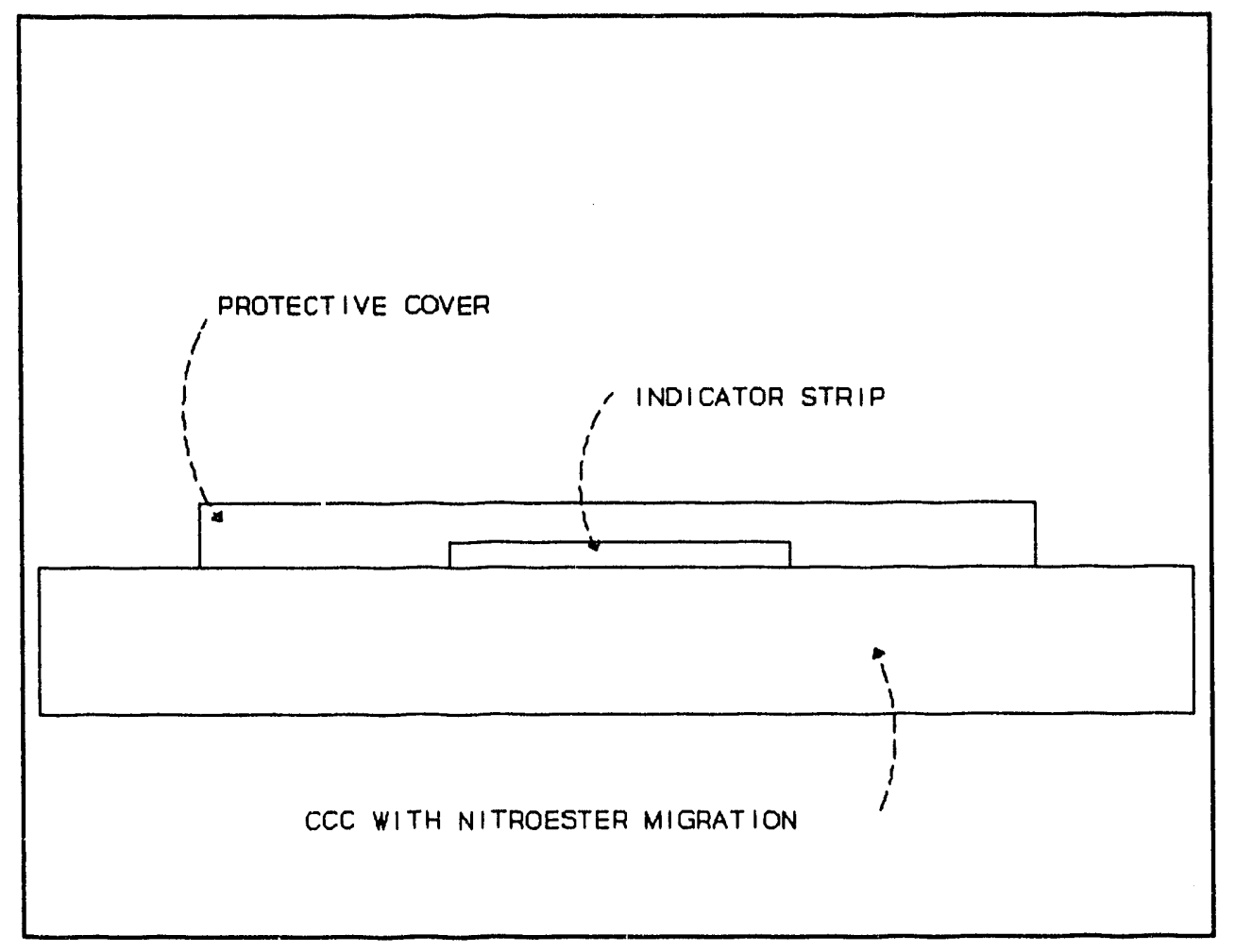

Figure 3. Diagram of Nitroester Indicator Structure.

\section{Results and Discussion}

\section{Indicator Design}

The indicator chosen is the modified Griess reagent that is normally used in solution to test for compounds which contain the nitro group $\left(-\mathrm{NO}_{3}\right)$. This reagent was the only chemistry located in our survey task (5) which satisfied the criteria of being compatible with ccc material and not requiring an auxiliary light source (e.g., UV) for color development or fluorescence detection. In its typical application, a sample is added to a solution of $\mathrm{N}$-(1-naphthyl)ethylenediamine dihydrochloride and sulfanilic acid. (This solution is the modified Griess reagent). Powdered zinc is added to the resulting solution. The zinc reduces nitrate to nitrite which reacts with the sulfanilic acid forming a diazo compound which then combines with the $\mathrm{N}-(1-$ naphthyl)ethylenediamine dihydrochloride to form a diazo dye. This reaction is diagrammed in Figure 4. 


$$
\mathrm{R}-\mathrm{O}-\mathrm{NO}_{2} \frac{\mathrm{Zn}}{\mathrm{H}^{+}} \longrightarrow \mathrm{HNO}_{2}
$$

Figure 4. Mechanism of the Modified Griess Reaction tsed for the Nitroester Indicator.

The choice of the medium used to support the indicator reagents affects the stability of the indicator. When sulfanilic acid solution was placed on common cellulose-based filter paper, after a short time the color of the paper changed to a pale yellow. It is likely that impurities in the cellulose reacted with the sulfanilic acid. Three other types of paper supports were tested with the modified Griess reagent solution: Whatman no. 40 ashless cellullose-based filter paper, Nucleopore prefilter paper (no. 211707), and Nucleopore glass fiber filter paper (no. 211825). After 17 days storage in the dark at room temperature the Whatman ashless filter paper turned purple, similar to the reaction with nitroester. The Nucleopore prefilter paper changed to a light gray, and no change was observed for the glass fiber-based paper. Thus, the Nucleopore glass fiber filter paper was used as the indicator support medium.

A protective coat or surface shield also is necesary to protect the reagents from contact with air and also from physical damage. several commercial household glues, polyurethane, epoxy, and plastic tapes were evaluated. Bel Art tape (described in more detail in the next chapter) was found to give the best protection for the nitroester indicator strip.

The stability of the components of the indicator reagent also is important to the lifetime of the indicator strip. Of the reagents used, the sulfanilic acid appeared to be the least stable. It was observed to decompose with time and change from white to pale yellow in color. The sodium 
salt should be more stable, so a test was made of its suitability to replace the free acid in the modified Griess reagent. Using the 2 layer reagent configuration, it did not permit a nitroesterspecific color change to be produced, and was judged unsuitable. Apparently, it cannot convert nitrite to nitrous acid, which is required for reaction with the sulfonic acid to form the diazonium salt. Additional work is needed to identify more stable reagents.

In early attempts to perfect a test strip, many different means of applying the modified Griess reagent and the powdered zinc to the indicator were tested. Experiments were conducted to apply a thin, even layer of zinc powder onto paper by spraying an aqueous zinc suspension. This approach was not successful. It was found that simply brushing the zinc on using a small brush was satisfactory. In one test, a glass fiber filter paper was wetted with the modified Griess reagent which was then dried by vacuum desiccation. Powdered zinc was then dusted on the reverse side of the filter paper. This test square was brought into contact with a surface containing a nitroester. If there was a sufficient quantity of the nitroester to migrate through the filter paper, it would readily yield a color change. However, these test squares were very sensitive to small amounts of nitroester and developed a significant color change over extended periods of time even in the absence of NG. The reagent also is light-sensitive, which adds to the blank problem. Test plates were normally wrapped in aluminum foil while waiting for color development to protect the reagents from photodegredation.

Frequently, test materials prepared as described immediately above would develop a slight color before applying the powdered zinc. Experiments were then conducted with the dry materials. At first, the modified Griess reagents were blended in a $1 / 1$ mole ratio. Fiberglass filter paper was dusted with zinc. A test strip of this zinc-coated filter paper was placed in contact with a test square containing NG. A small portion of the dry Griess reagent mix was placed on the surface of the test strip and the entire unit then sealed against a glass plate for color tests. This is the general preparation method used for the performance tests described below.

\section{Performance of Nitroester Indicator Strip Designs}

The performance of three reagent configurations was tested. A nitroester test was started on 6-8-92 for the configuration with zinc powder on the back side of the indicator strip, and the modified Griess reagent [ $\mathrm{N}$-(1-naphthyl)ethylenediamine dihydrochloride plus sulfanilic acid] on the top side. The reason for placing the zinc powder behind the strip was to prevent the dark color of the powder from obscuring the visual detection of the colored dye. In this configuration, the nitroesters are reduced to nitrite on the bottom of the strip, and the nitrite then diffuses into the Griess reagent on the top of the strip to form the colored diazo dye. The configuration of the test plate in Figure 5 is listed in Table 1. 
Table 1. Relative Positions and Nitroester Concentrations of Test Rectangles in Figure 5.

\begin{tabular}{|c|c|c|}
\hline \multicolumn{3}{|c|}{ Relative Position and Nitroester Concentration, $\mathrm{mg} / \mathrm{g}$} \\
\hline 3 & 0 (Blank) & 3 \\
\hline 6 & 0 (Blank) & 6 \\
\hline 9 & - & 9 \\
\hline
\end{tabular}




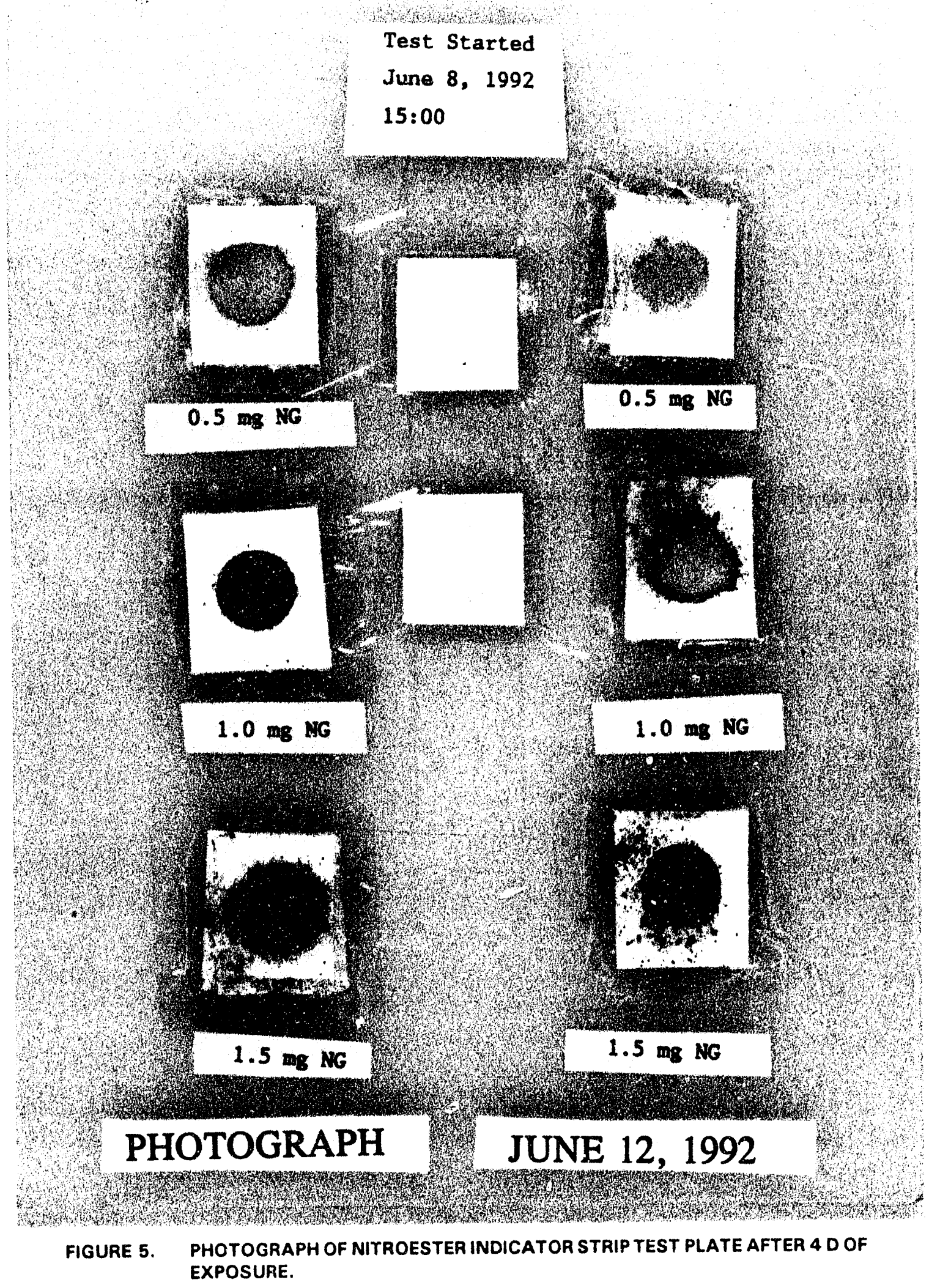


The photograph in Figure 5 was taken after $4 \mathrm{~d}$ of exposure (6-12-92). This test shows that color development was rapid and the color density was proportional to the NG concentration. The blanks had only a slight discoloration and were not significant in relation to the other test strips.

The performance of the 3 reagent configurations was demonstrated in a simultaneous test. The test plate was similar in makeup to the above. The first configuration had the $\mathrm{N}-(1$ naphthyl)ethylenediamine dihydrochloride on the surface of the indicator strip with a mixture of the sulfanilic acid and zinc powder on the reverse side. The second configuration had the dry modified Griess reagent on the surface of the indicator strip with zinc powder on the reverse side. The final configuration had a mixture of the dry modified Griess reagent on top of the zinc powder, all on the surface of the indicator strip. Table 2 diagrams the relative positions and configurations of the indicator strips on the test plates shown in Figures 6 and 7.

Table 2. Relative Positions, Reagent Configurations and Nitroester Concentrations for Test Plate in Figures 6 and 7.

\begin{tabular}{|c|c|c||}
\hline \multicolumn{3}{|c||}{$\begin{array}{c}\text { Relative Position for } \\
\text { Concentration of Nitroester, mg/g } \\
\text { Reagent Configuration }\end{array}$} \\
\hline $\begin{array}{c}0 \text { (Blank) } \\
\mathrm{A} / \mathrm{B}+\mathrm{C}\end{array}$ & $\begin{array}{c}0 \text { (Blank) } \\
\mathrm{A}+\mathrm{B} / \mathrm{C}\end{array}$ & $\begin{array}{c}0 \text { (Blank) } \\
\mathrm{A}+\mathrm{B}+\mathrm{C} /\end{array}$ \\
\hline 0.6 & 0.6 & 0.6 \\
$\mathrm{~A} / \mathrm{B}+\mathrm{C}$ & $\mathrm{A}+\mathrm{B} / \mathrm{C}$ & $\mathrm{A}+\mathrm{B}+\mathrm{C} /$ \\
\hline 3 & 3 & 3 \\
$\mathrm{~A} / \mathrm{B}+\mathrm{C}$ & $\mathrm{A}+\mathrm{B} / \mathrm{C}$ & $\mathrm{A}+\mathrm{B}+\mathrm{C} /$ \\
\hline 13 & 13 & 13 \\
$\mathrm{~A} / \mathrm{B}+\mathrm{C}$ & $\mathrm{A}+\mathrm{B} / \mathrm{C}$ & $\mathrm{A}+\mathrm{B}+\mathrm{C} /$ \\
\hline
\end{tabular}

$A=\mathrm{N}-(1-$ naphthyl)ethylenediamine dihydrochloride

$\mathrm{B}=$ Sulfanilic acid

$\mathrm{C}=$ Powdered zinc

Photographs of the test plate after $1 \mathrm{~d}$ of exposure (Figure 6) showed a marked color change for the $13 \mathrm{mg} / \mathrm{g}$ nitroester test squares for all 3 reagent configurations. By $16 \mathrm{~d}$ of exposure (8-20-92, Figure 7) there were significant color changes at all concentrations of nitroester. The configuration with all 3 reagents present on the surface of the indicator strip gave the 


\section{Nitro Ester Test}

8-4-92
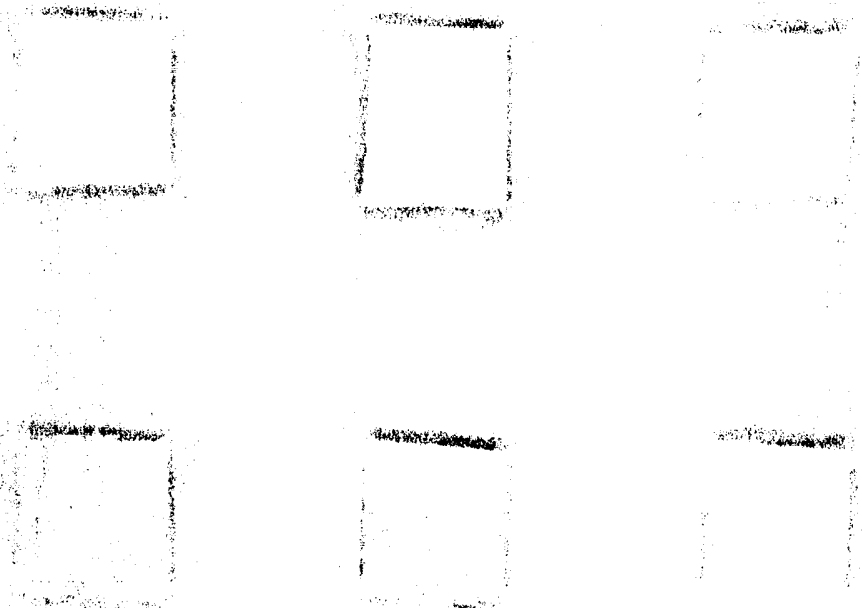

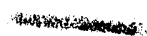
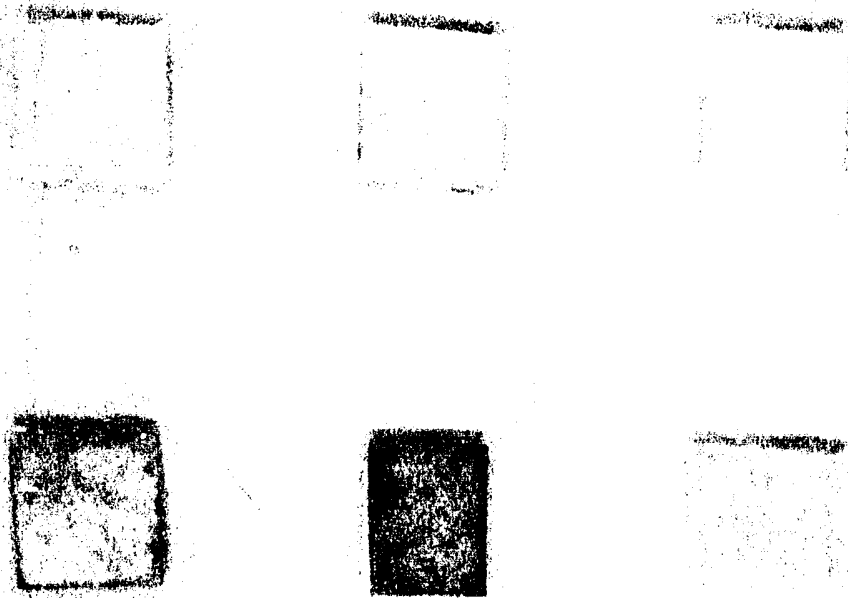

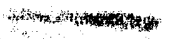
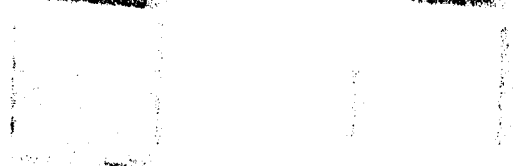

FIGURE 6. PHOTOGRAPH OF NITROESTER INDICATOR STRIP TEST PLATE AFTER 1 D OF EXPOSURE. 


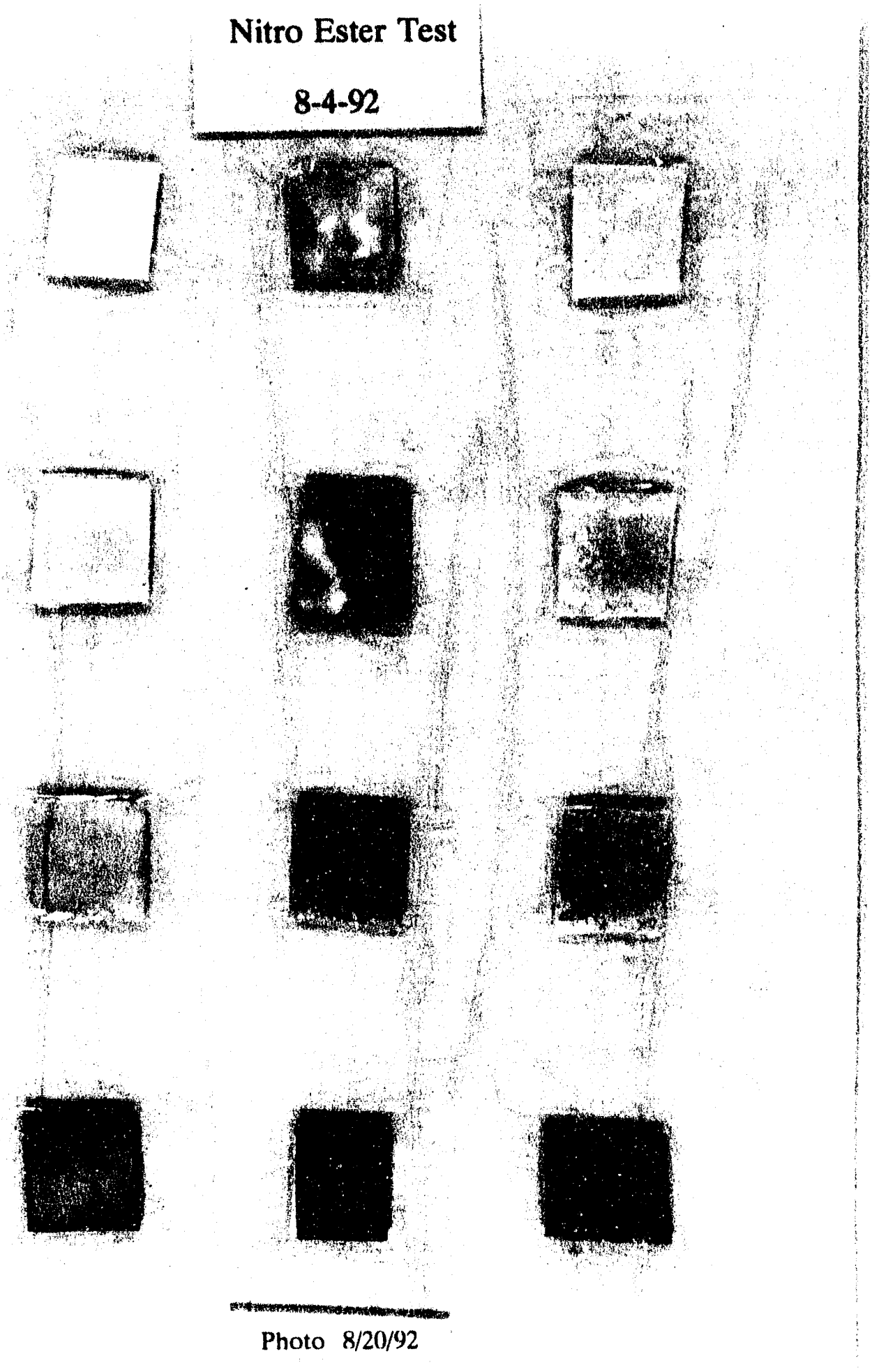

FIGURE 7. PHOTOGRAPH OF NITROESTER INDICATOR STRIP TEST PLATE AFTER $16 \mathrm{D}$ OF EXPOSURE. 


\section{MOISTURE INDICATOR}

\section{Background}

Moisture accumulation in ecc munitions, from exposure to very wet environments or from the round actually lying in a pool of water, causes the case wall to soften and weaken, and the adhesive joint tensile strength to decrease (4). Visual indicators would allow the identification of individual rounds which have accumulated moisture, and which could be removed from deployment for drying out.

As noted in our previous report (5), the ideal moisture indicator strip should meet several requirements:

a. The indicator strip must be designed for placement on the surface of the ccc wall and indicate the level of moisture in the casing. It should not interfere with the storage, chambering, or firing of the round.

b. The color change must be readily visible.

c. The chemistry of the indicator must be compatible with the ccc, and not cause deterioration of the cec. It also must not pose a hazard to gun crews.

d. The indicator strip should have a long lifetime (decades).

e. The indicator strip reaction should be reversible. (If the strip indicates high content of moisture the round is transported to a drying area where moisture is to be removed. Indicator color should reverse, indicating the removal of the moisture.)

The target moisture concentration to be detected by indicator strips was estimated from previous ccc coupon experiments $(4,5)$. Uncoated ecc coupons (cut from only the nitrocellulose/wood fiber portion of the M829 case wall, and not ircluding the cellulose adapter) equilibrated with ambient laboratory air (ca. $45-65 \% \mathrm{RH})$ to a moisture content of $4.4 \pm 0.51 \%(\mathrm{n}=3)$. When exposed to air saturated with water vapor, the moisture content increased to $13.0 \pm 0.15 \%$ (n $=3$ ). As shown in Figure 8, this process reached a value close to equilibrium within $7 \mathrm{~d}$ of exposure. The reason for the drop in moisture at $d 11$ is not clear. The results for the longer exposures suggest a slow climb to a slightly higher degree of hydration by $28 \mathrm{~d}$. This behavior suggests that more than one mechanism for hydration or water uptake is present. For example, the cellulose sites in the nitrocellulose/cellulose matrix may take up water much more rapidly than do the nitrocellulose, which could lead to a rapid initial uptake of moisture, and then a slower uptake by the lower aftinity nitrocellulose. Significant decreases in case wall indentation hardness (10\% decrease) and adhesive joint tensile strength (39\% decrease) were observed (4) in ccc coupons exposed to air at $21^{\circ} \mathrm{C} / 97 \% \mathrm{RH}$ for $29 \mathrm{~d}$. Moisture effects appeared to be reversible upon drying, if an elevated temperature were not present during the exposure to water vapor. When the coupons were soaked in water, the cec coupon moisture concentration increased to $20.2 \pm 2.8 \%(n=3)$. This hydration required 4 to $8 \mathrm{~d}$ to reach an apparent equilibrium, as depicted in Figure 9. After $17 \mathrm{~d}$ of soaking, case wall indentation hardness decreased by $14 \%$, and the adhesive joint tensile strength dropped by $44 \%$. A separate 
experiment for the infrared spectroscopy test (see later chapter) yielded a $32 \%$ moisture content, but only one replicate was run. These results suggest that a 20 to $30 \%$ moisture content can be reached by ecc material, but effects can be observed with moisture concentrations of $13 \%$. Allowing a conservative factor of 2 or 3 for the indicator strip, target moisture concentrations of ca. $10 \%$ appear desirable. Accordingly, the range of moisture concentrations used in the indicator strip tests approached $10 \%$.

\section{Experimental}

Two forms of moisture indicators were developed. One was based upon glass fiber paper saturated with cupric chloride $\left(\mathrm{CuCl}_{2}\right)$, and the other was based upon cupric chloride pellets.

\section{Impregnated Glass Fiber Indicator}

For the glass fiber based indicator, Nucleopore glass fiber paper with a $7 \mu \mathrm{m}$ porosity (part number 211825 ) was saturated with cupric chloride by soaking $3.8 \mathrm{~cm} \times 7.6 \mathrm{~cm}(1.5$ in 33 in) strips in concentrated cupric chloride solution $\left(10 \mathrm{~g}\right.$ of $\mathrm{CuCl}_{2} \cdot 2 \mathrm{H}_{2} \mathrm{O}$ per $100 \mathrm{~mL}$ of water $)$ and drying overnight at $110^{\circ} \mathrm{C}$. During the drying step the salt was dehydrated $\left(\mathrm{CuCl}_{2}\right)$. The dry, dehydrated strips were cut into smaller squares or rectangles for testing, and were sealed to the test squares with tape to prevent atmospheric moisture from interfering with the response.

\section{Pelletized Indicator}

300 to $400 \mathrm{mg}$ of $\mathrm{CuCl}_{2}$ were pressed into a thin pellet using a $1.27 \mathrm{~cm}(0.5 \mathrm{in})$ pelletizer at $10,335 \mathrm{kP}_{\mathrm{a}}(1,500 \mathrm{psi})$ for $30 \mathrm{sec}$. The pellets were placed in an oven held at $100^{\circ} \mathrm{C}$ for redrying and storage. They were supported by placing in a cardboard strip with a round hole cut to the same diameter as the pellet. 


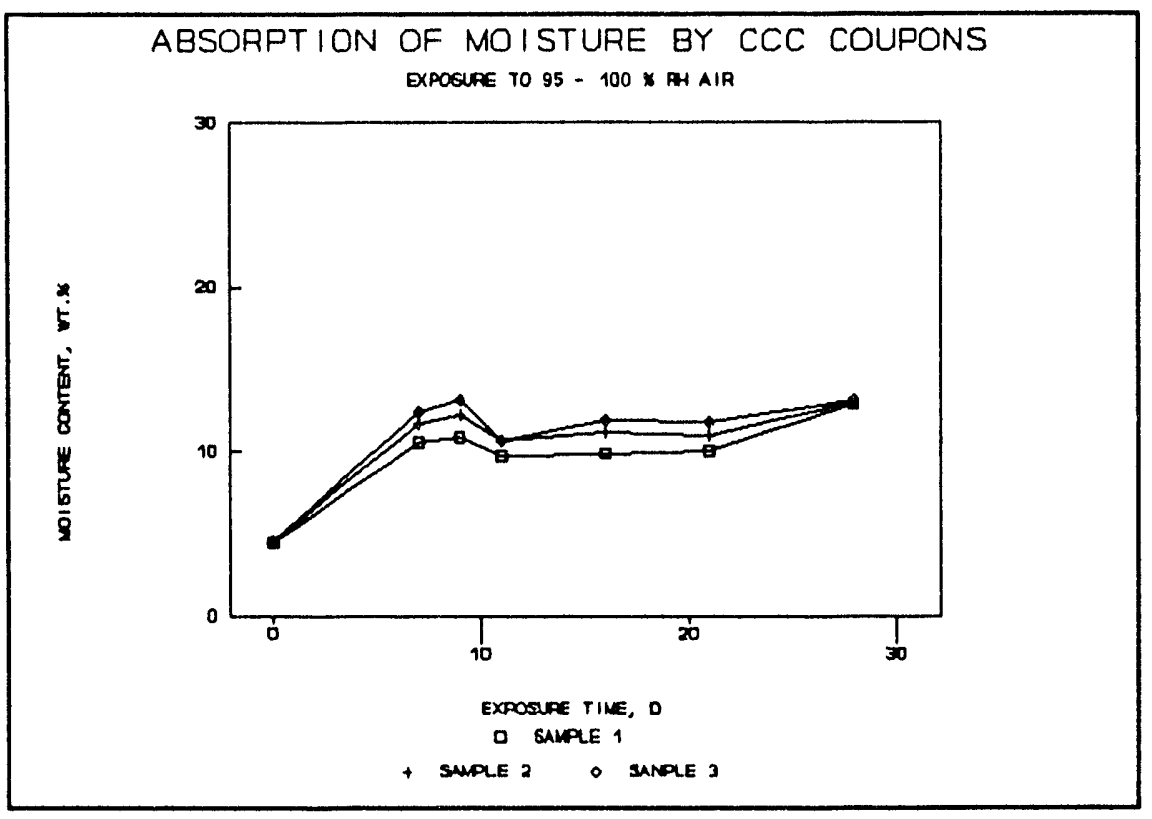

Fig. 8. Uptake of Moisture by CCC Exposed to $95-100 \%$ RH Air.

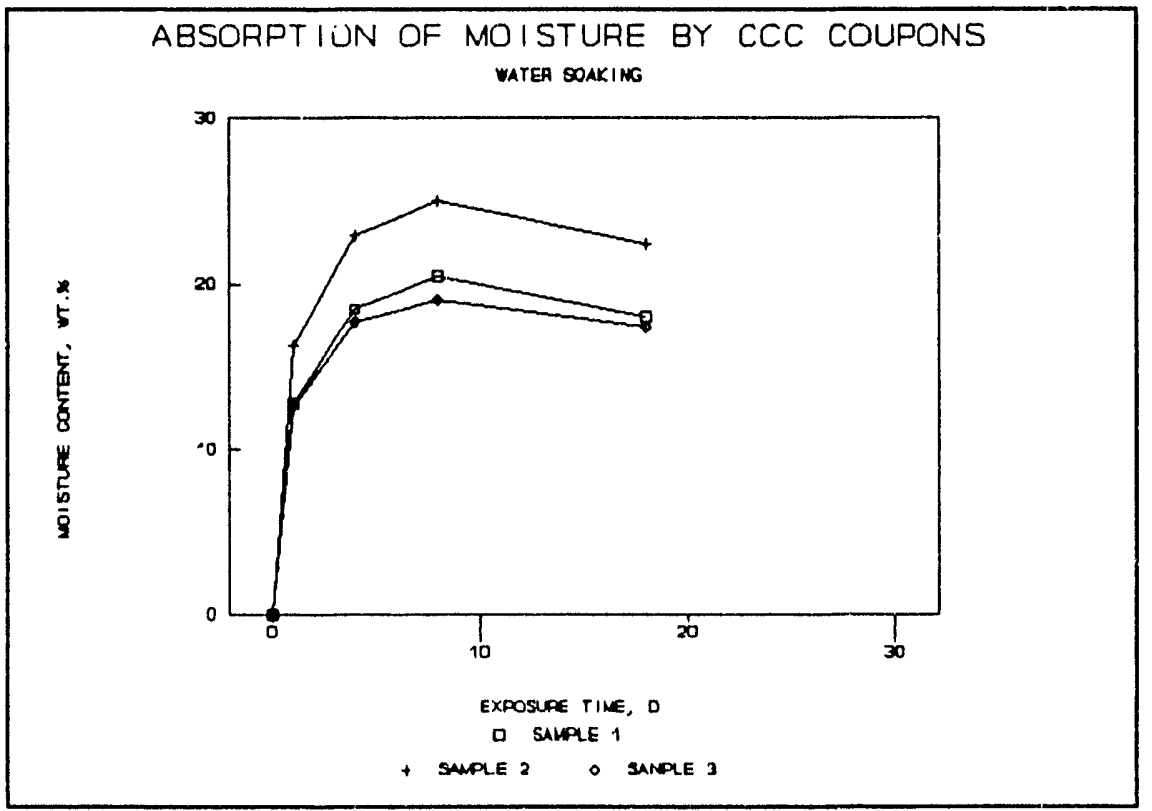

Fig. 9. Moisture Uptake by CCC Placed in Water. 


\section{General Design of Indicator Strips}

In contrast to the nitroester indicator, it was necessary to place a semipermeable barrier between the indicator and the test sample to potentiate the indicator response. Accordingly, the test sample was covered with plastic tape. To provide a path for the moisture to diffuse to the indicator, small holes (ca. 20 per indicator strip) were punched in the tape, using the point of a pencil compass. The indicator, consisting of either the impregnated paper or the salt pellet supported in a cardboard square, was then sealed onto the test strip using adhesive tape to prevent uptake of atmospheric moisture by the indicator. The structure of the moisture indicator strip is diagrammed in Figure 10.

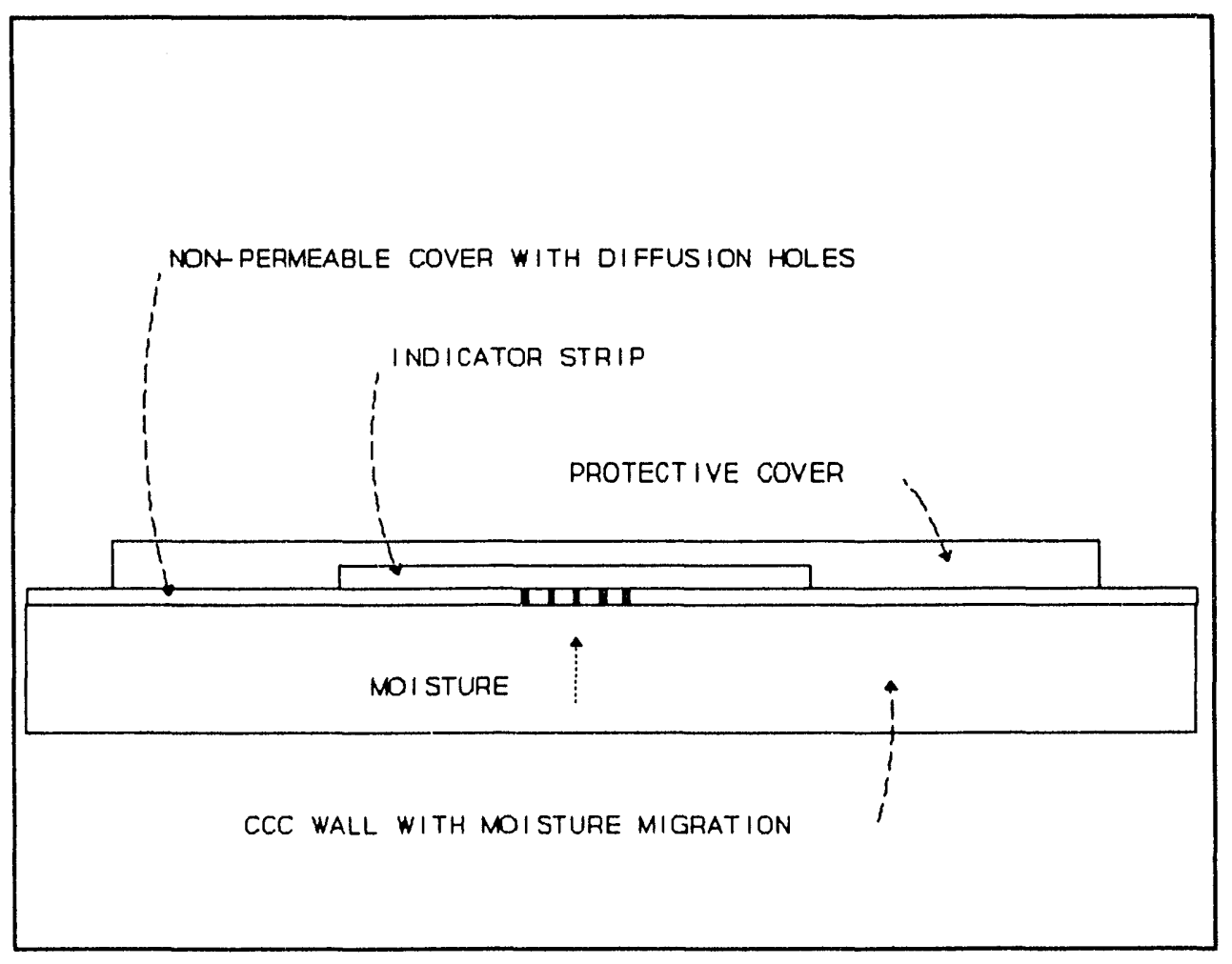

Fig. 10. Diagram of Moisture Indicator Design. (Relative thicknesses are not shown to scale.) 


\section{Results and Discussion}

\section{Indicator Design}

Indicators are compounds that change color as they become hydrated. The chosen moisture indicator compound, due to its marked color change, is cupric chloride. The molecular weight of $\mathrm{CuCl}_{2}$ is 134 , and in the presence of moisture it hydrates to $\mathrm{CuCl}_{2} \cdot 2 \mathrm{H}_{2} \mathrm{O}$ (molecular weight 170). This means that $1 \mathrm{~g}$ of the dry $\mathrm{CuCl}_{2}$ will absorb $0.27 \mathrm{~g}$ of water. In the dry form the cupric chloride is brown but it turns to a blue-green when hydrated. It is a stable, noncorrosive salt which should not damage cec material.

These salts are able to draw moisture from a contacted source having a very low concentration of moisture. If that moisture is replaced or the source has sufficient moisture to completely hydrate the salt the color change will develop in time. Naturally, the color change is slower if the moisture content is low or if a large quantity of the cupric chloride is employed.

During earlier testing of the copper salt, solutions of the salt in water were prepared and test strips were made by wetting glass fiber filter papers with the solution and drying these test strips in an oven. It was found that strips with very low concentrations of the salt were much too sensitive and would change color when contacted with very low concentrations of moisture in the test material. Filters with very high concentrations of the salt were less sensitive but also became hydrated and changed color, although over a longer time period. We finally began pressing pellets of the dry salt. Between three and four hundred milligrams of the salt are pressed in a $1.27 \mathrm{~cm}\left(0.5 \mathrm{in}\right.$ ) pelletizer at $10,335 \mathrm{kP}_{\mathrm{u}}$ (ca. $1,500 \mathrm{psi}$ ). These pellets, containing much more cupric chloride than can be evaporated on a small filter square, required much more moisture to effect a color change. We are able to attach these pellets to a surface and make simple estimates of the moisture concentration based upon the time required for the color change to take place.

The material used to achieve a seal of the test material is quite important to prevent atmospheric moisture from contaminating the indicator and to potentiate the diffusion of moisture from the test rectangle into the indicator. Some plastic tapes, such as $3 \mathrm{M}$ Prescription Label Tape were too permeable to moisture. An early sample of what appears to be Bell Arts Lab Label Tape provided the best protection, and the second best tape was from a recent lot of the Bell Arts Lab Label Tape. However, the latter had a slightly different colored tinge on the roll than did the former, suggesting a difterent formulation. Clear Scotch mailing tape and $3 \mathrm{M}$ Highland Brand 371 clear tape also were good. The tape seals well if smoothly applied. It is somewhat difficult to make a smooth seal if the test square is very thick. In cases where wrinkles in the tape seal might transmit moisture, a cross-seal with the same tape is necessary.

\section{Performance of The Impregnated Glass Fiber Indicator}

The performance of the impregnated glass fiber indicator was tested using cellulose rectangles having known moisture contents. To record the color change dependency upon moisture concentration and time, photographs were taken of the test plates at various intervals. 
Test rectangles $(3.81 \mathrm{~cm} \times 5.08 \mathrm{~cm}$, or 1.5 in $\times 2$ in) were cut from matting board and dried in an oven at $100^{\circ} \mathrm{C}$. The rectangles with $0 \%$ moisture were used as removed from the oven. Other rectangles were weighed after removal from the oven, and were exposed to the atmosphere to absorb moisture. They were reweighed to determine the moisture gained. Test rectangles with moisture concentrations of $3.5 \%, 5.3 \%$, and $9 \%$ were prepared. The test rectangles were sealed in clear plastic tape to prevent further moisture uptake. An indicator strip of the cupric chloride impregnated glass fiber was mounted on the surface of each test rectangle. To permit moisture from the test square to migrate into the indicator strip, small holes were punched through the tape seal. The fine tip of a drafting compass was used to form these holes ( 20 holes per test rectangle). Clear plastic tape was used to seal the indicator strip to the test rectangle, thus preventing ambient moisture from reaching the indicator and causing a false response. Table 3 shows the relative positions of the test strips and their moisture concentrations for the test plates in Figures $11-14$.

Table 3. Relative Positions of Test Strips in Figures $11-14$ and Their Moisture Concentrations.

\begin{tabular}{|c|c|c|c|}
\hline \multicolumn{4}{|c|}{$\begin{array}{l}\text { Relative Position and Moisture Concentration, \% } \\
\text { (Date of Preparation) }\end{array}$} \\
\hline $\begin{array}{c}0 \\
(4-27)\end{array}$ & $\begin{array}{c}3.53 \\
(4-27)\end{array}$ & $\begin{array}{c}5.25 \\
(4-27)\end{array}$ & $\begin{array}{c}9.02 \\
(4-27)\end{array}$ \\
\hline $\begin{array}{c}0 \\
(4-28)\end{array}$ & $\begin{array}{c}3.53 \\
(4-28)\end{array}$ & $\begin{array}{c}5.25 \\
(4-28)\end{array}$ & $\begin{array}{c}9.02 \\
(4-28)\end{array}$ \\
\hline
\end{tabular}

The test plate photograph taken on 5-1-92 (Figure 11) shows that in only $4-5 \mathrm{~d}$ the indicators mounted on the test rectangles containing $9 \%$ moisture have already begun to change. The difference in intensity of color development between the 4-27 and the 4-28 samples is probably due to different masses of cupric chloride impregnated in the indicator strips. The photo of 5-792 (Figure 12) shows that in $10-11 \mathrm{~d}$ the $5 \%$ moisture test squares are starting to change and that by $18-19 \mathrm{~d}$, on 5-19-92 (Figure 13), all the strips have started color development. By 84 - $85 \mathrm{D}$ of exposure, on 7-21-92 (Figure 14), all the indicators had completely changed indicating complete hydration of the cupric chloride. The response to the $0 \%$ moisture test rectangle suggests that either very small traces of moisture remained in the cellulose matts after drying, or, more likely, that the plastic tape outer cover has a very low permeability to moisture which permitted atmospheric moisture to diffuse into the strip.

The response of this design of the impregnated glass fiber indicator strip thus is a temporal function, in that the time required for color development indicates the moisture concentration 


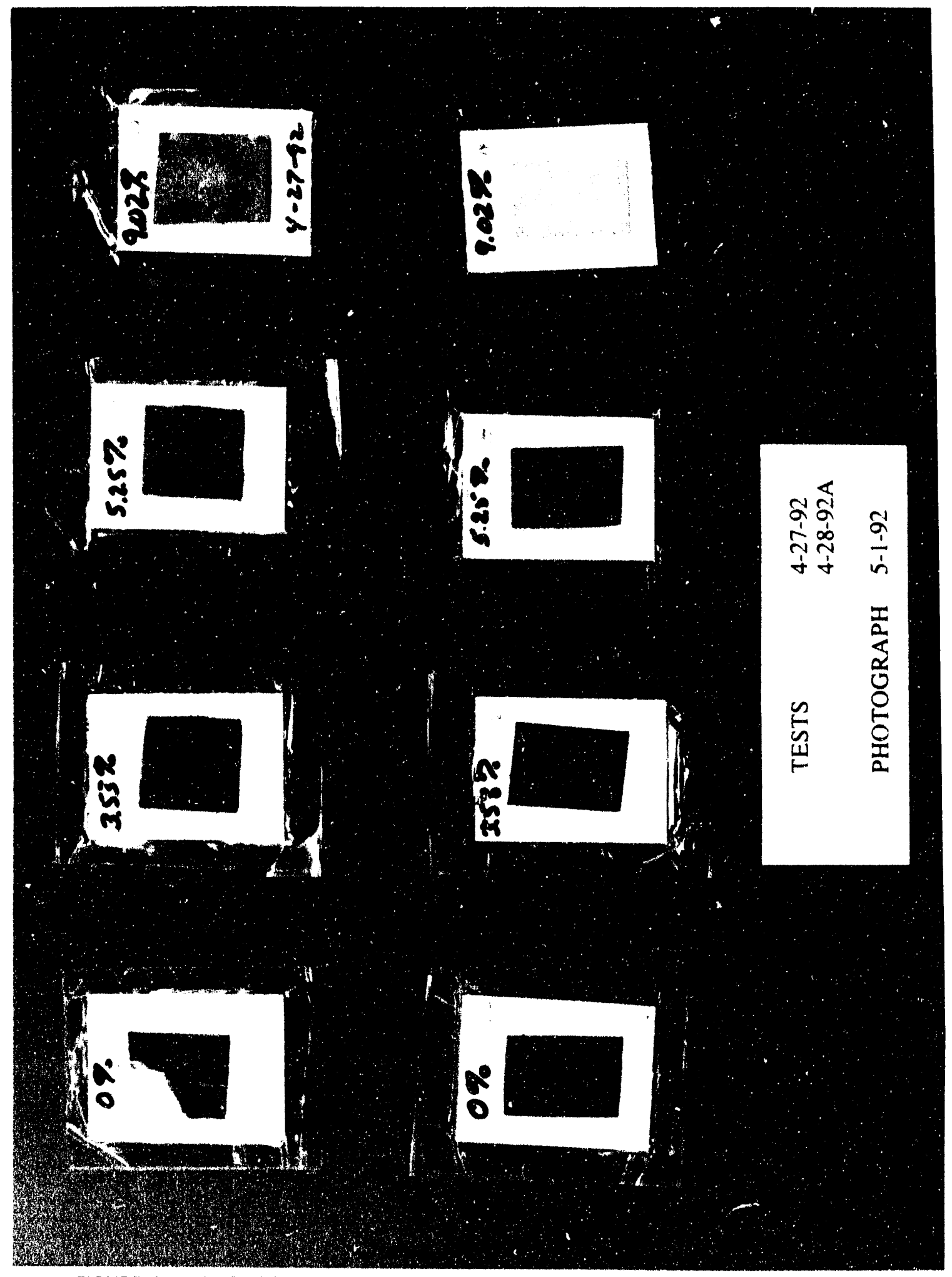

FIGURE 11. PHOTOGRAPH OF MOISTURE INDICATOR TEST PLATE AFTER 4 - 5 D OF EXPOSURE. 


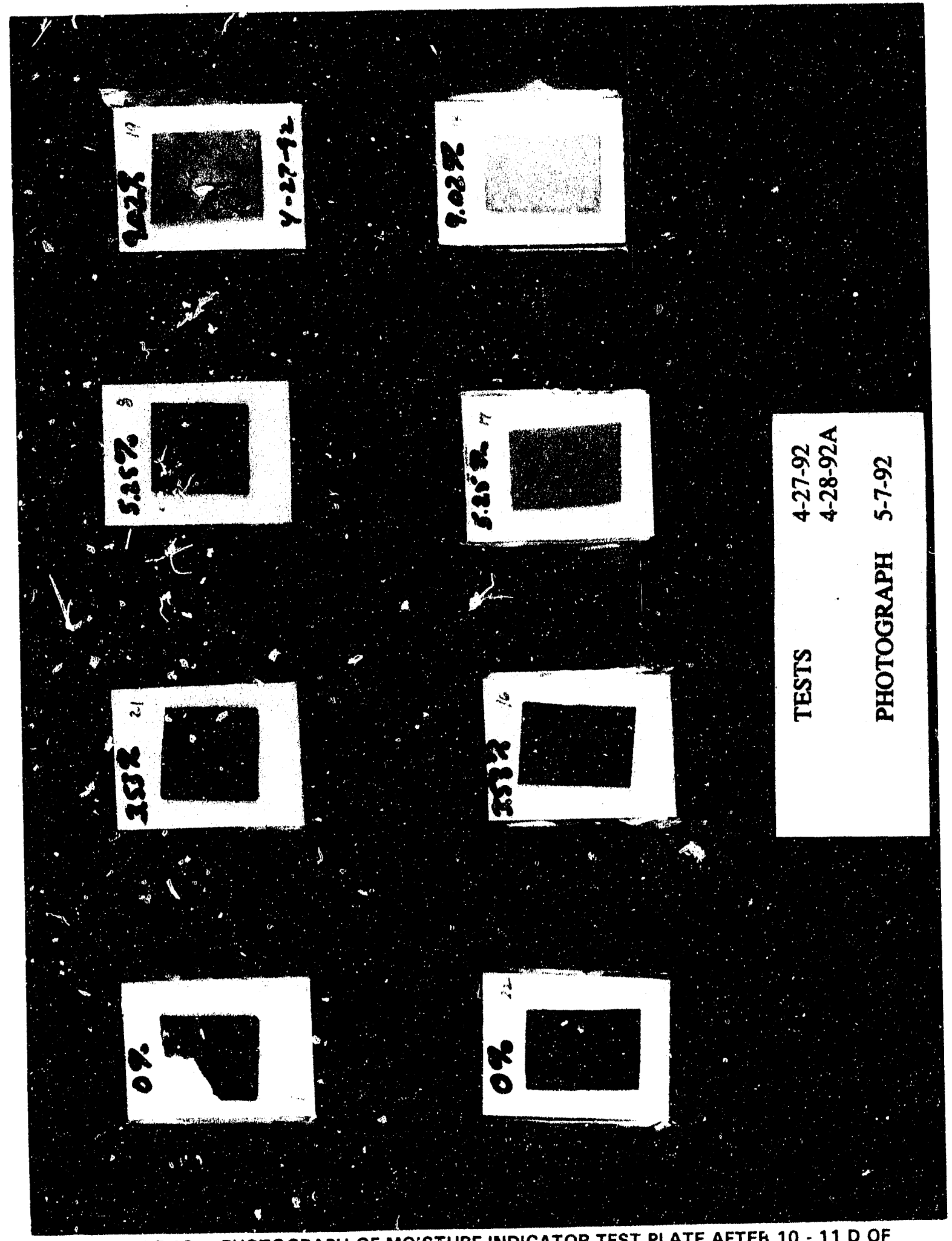

FIGURE 12. PHOTOGRAPH OF MOISTURE INDICATOR TEST PLATE AFTEF 10 - 11 D OF EITPOSURE. 


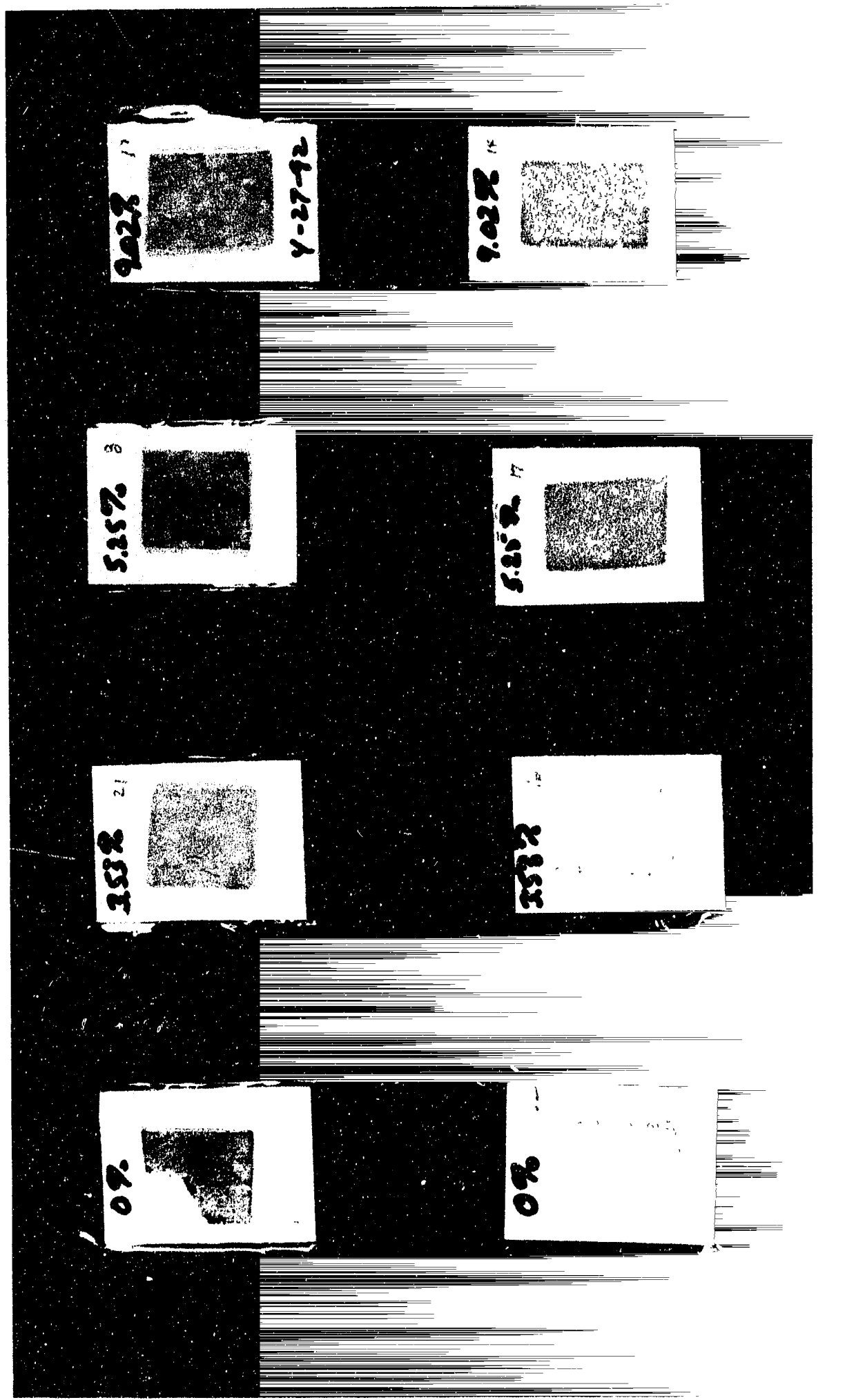

FIGURE 13. PHOTOGRAPH OF MOISTURE INDICATOR TEST PLATE AFTER EXPOSURE. 


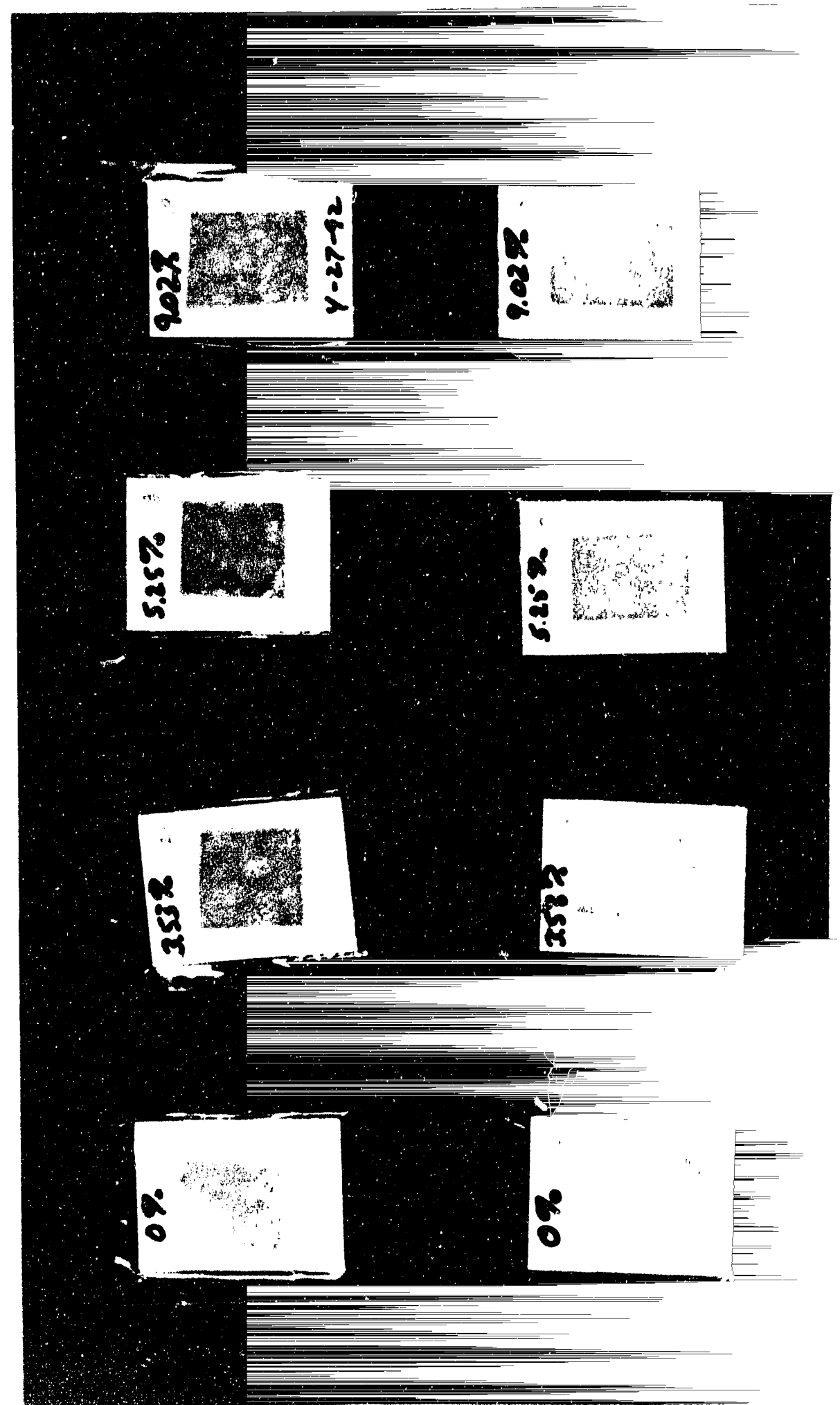

FIGURE 14. PHOTOGRAPH OF MOISTURE INDICATOR TEST PLATE AFTER 8 EXPOSURE. 
of the test rectangle. Discrimination power appears to be limited to concentrations of moisture above ca. 5 to $9 \%$.

\section{Performance of The Pelletized Indicator}

As noted above, the sensitivity of the impregnated glass fiber indicator led to the development of the pelletized indicator design. The indicator performance test was conducted similarly to that for the impregnated glass fiber indicator. Test squares of cellulose matting board were prepared by drying at $100^{\circ} \mathrm{C}$ and allowing moisture to be absorbed in the matting material. The mass change in the test square determined the moisture content. The test squares were then sealed in the $3 \mathrm{M}$ Highland Brand clear mailing tape. Pellets mounted in cardboard squares were sealed on the surface of the test squares immediately after holes were pierced in the test covering to permit moisture migration from the test square into the pellet. Table 4 shows the relative positions and moisture concentrations of the test strips.

Table 4. Relative Positions and Moisture Concentrations of Test Strips in Figures $15-17$.

\begin{tabular}{|c|c|}
\hline \multicolumn{2}{|c|}{ Relative Position and Moisture Content, \% } \\
\hline 0 & 0 \\
\hline 2.7 & 2.8 \\
\hline 6.3 & 6.4 \\
\hline 8.0 & 8.1 \\
\hline
\end{tabular}

Photos taken of the test plates on 7-21-92, 8-4-92, and 8-20-92 are shown in Figures $15-17$. By $5 \mathrm{~d}$ of exposure (Figure 15, 7-21-92), the indicators for the $8 \%$ and $6 \%$ moisture test squares have considerably changed, and the $0 \%$ and $2.7 \%$ moisture tests showed no change. By $19 \mathrm{~d}$ of exposure (Figure 16, 8-4-92), the 6\% and greater moisture tests showed nearly complete color change but the $2.6 \%$ or less moisture tests showed no change. By $35 \mathrm{~d}$ (Figure $17,8-20-92$ ) the $2.7 \%$ test showed only slight changes around the edges, which may not be readily visible in the photo. With longer exposure time, the color would be expected to develop. It is important to note that over the test period, the $0 \%$ moisture test square did not change color. The pelletized indicator thus appears to provide better performance than does the impregnated glass fiber indicator.

By controlling the pellet size carefully and empirically determining a time vs. moisture concentration factor this indicator technique could be used to determine the moisture concentration of a ece wall casing. The polyurethane coating of the round would 

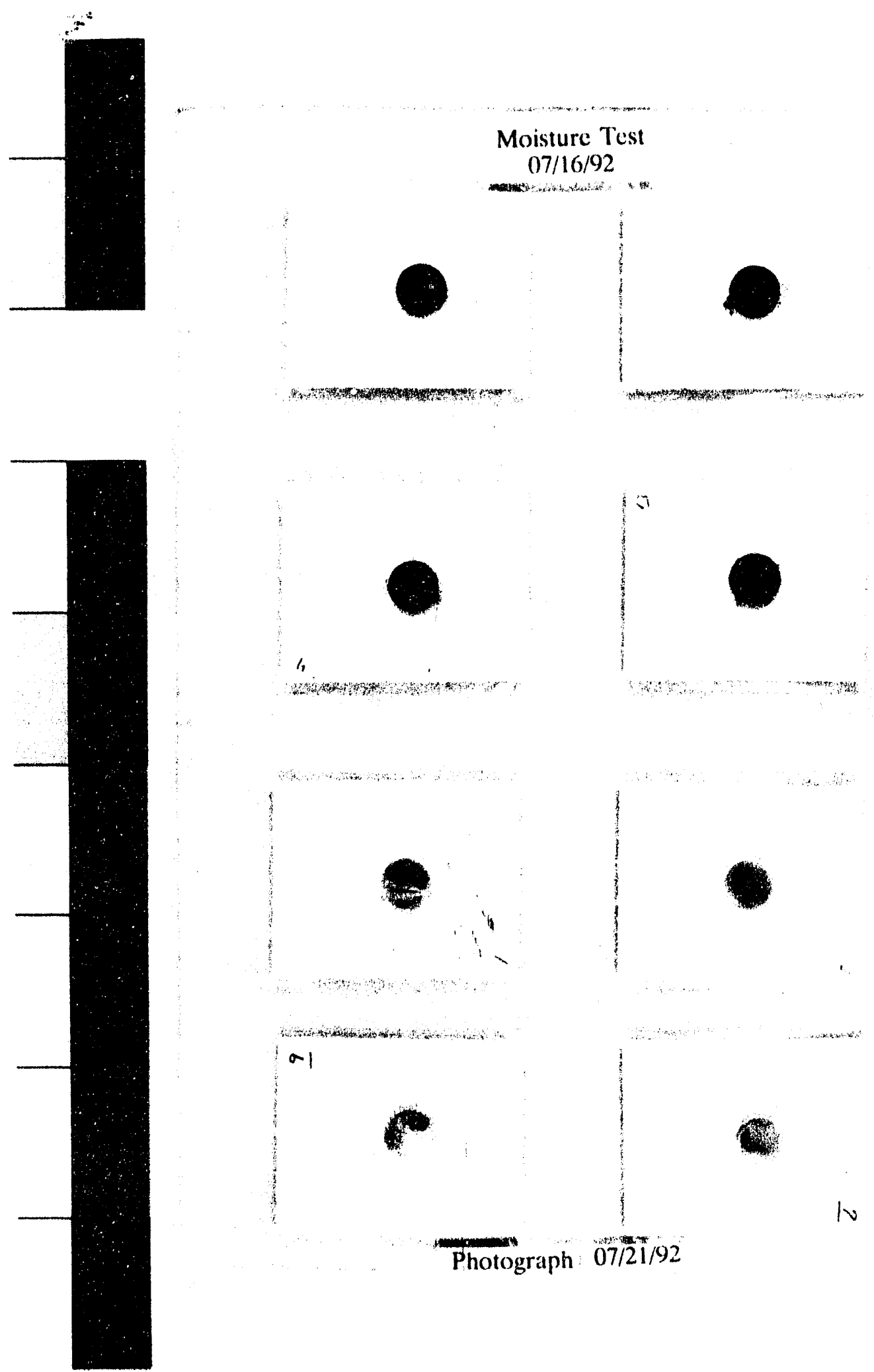

al

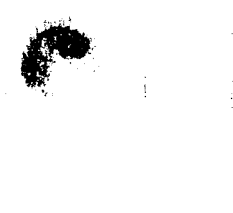

Photograph $07 / 21 / 92$

1v

FIGURE 15. PHOTOGRAPH OF MOISTURE INDICATOR TEST PLATE AFTER 5 D OF EXPOSURE. 
Moisture Test

$07 / 16 / 92$
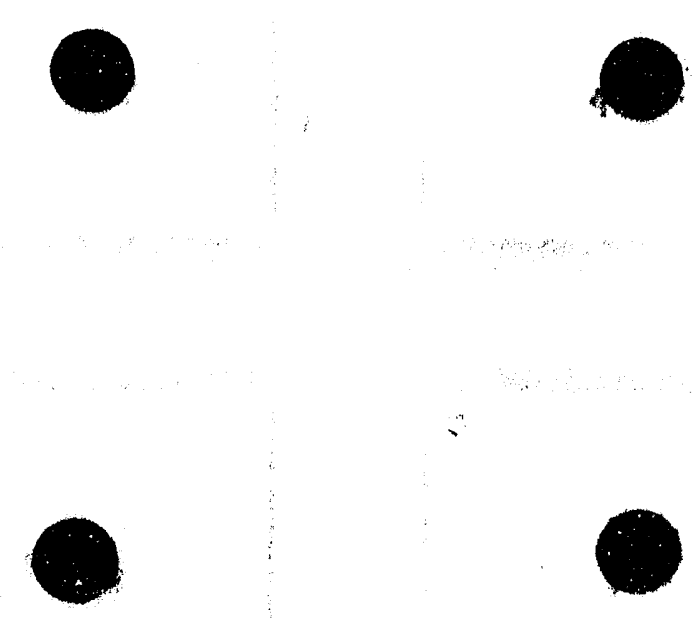

l,

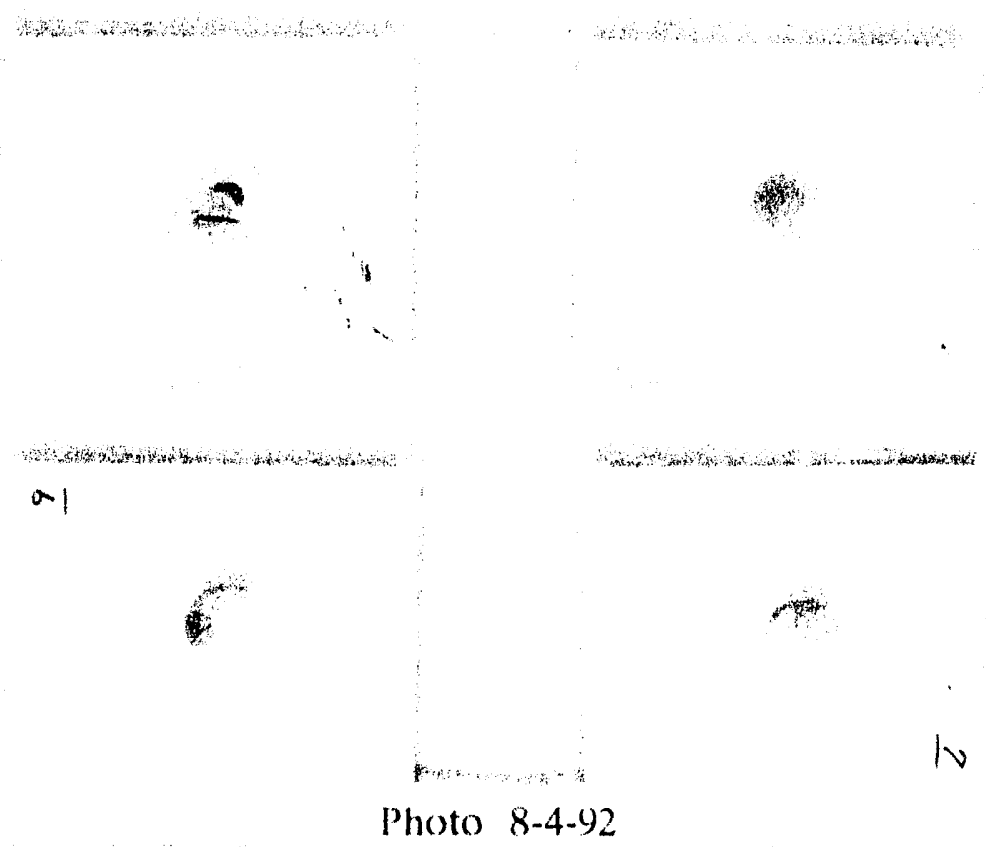

FIOURE 16. PHOTOGRAPH OF MOISTURE INDICATOR TEST PLATE AFTER 19 D OF EXPOSURE. 


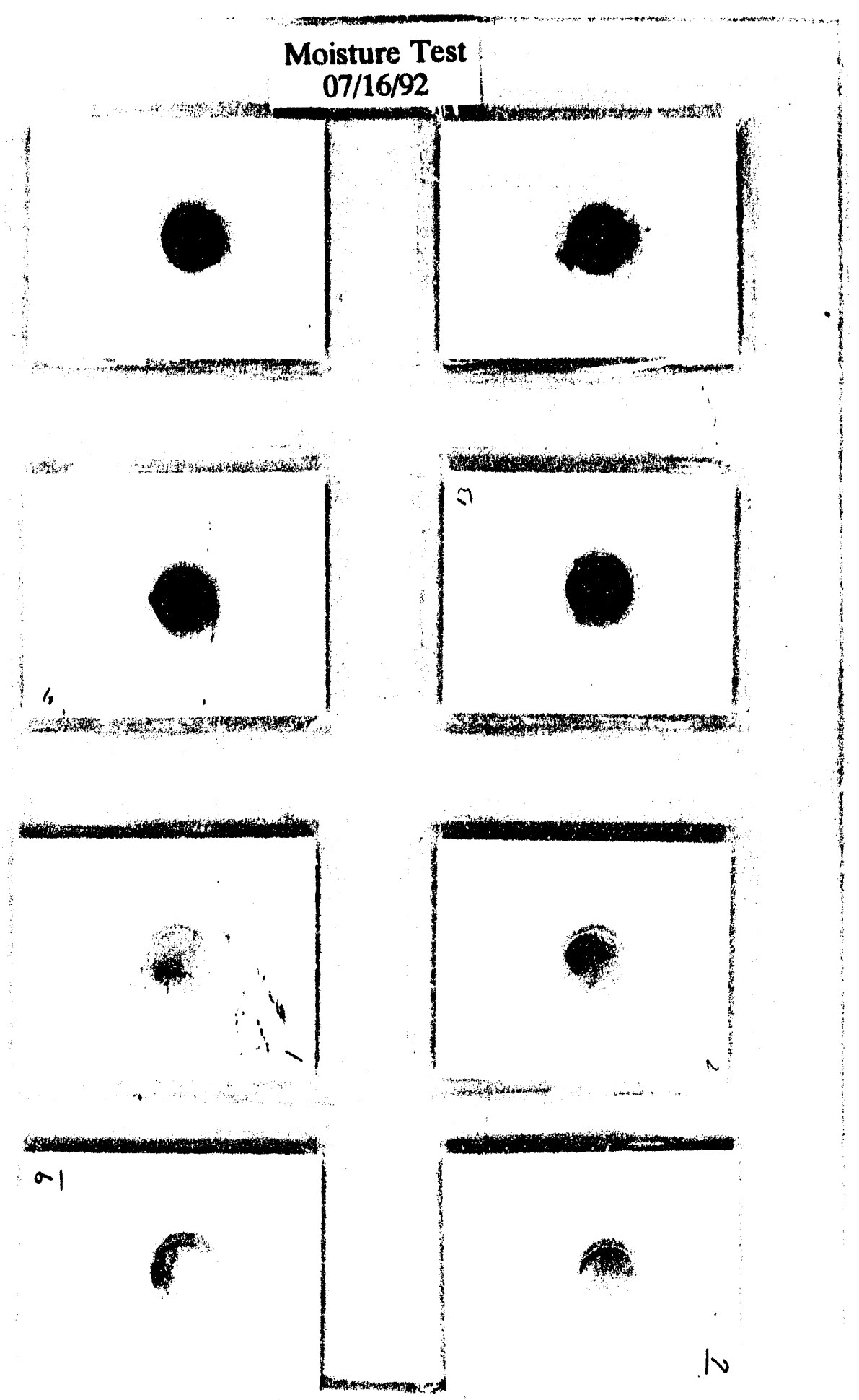

Photo $8 / 20 / 92$

FIGURE 17. PHOTOGRAPH OF MOISTURE INDICATOR TEST PLATE AFTER 35 D OF EXPOSURE. 
need to be pierced to permit moisture migration into the pellet. This indicator color change would not be reversible in this application as it would not be safe to heat the round to $100^{\circ} \mathrm{C}$ as is required to drive off the water of hydration in the hydrated cupric chloride.

\section{Conclusions and Recommendations}

The pelletized indicator is a better design than is the impregnated glass fiber indicator. Both are limited at the present to short-term indication (ca. 1 month) if the moisture content of the test material is above ca. 6\%. In its present state of development, the indicator also is not reversible on a practical basis. Clearly much additional development work would be necessary to improve its performance and to demonstrate its performance with actual ccc munitions. Some of the factors requiring work include development of a better potentiating barrier between the indicator and the cec to increase the useful lifetime of indication, a more durable and less permeable outer covering, development of methodology to apply the indicator to a ccc, and calibration of the indicator response to the actual case wall moisture. 


\title{
MOISTURE DETERMINATION BY DIELECTRIC MEASUREMENTS
}

\author{
Introduction
}

This chapter describes a preliminary evaluation of moisture determination in ccc munitions materials by measurements of the dielectric properties (capacitance) and conductivity (dissipation factor) or associated electrical impedance of the ccc as a function of frequency. The ultimate goal of this investigation was: (a) evaluation of commercially available portable instruments for non-destructive testing of ccc munitions for moisture accumulation, and (b) recommendation of instrument modifications needed for laboratory testing and field verification with munitions.

There is substantial technology available for moisture detection specifically by dielectric measurements. Advances in electronic instrumentation make this technology feasible and attractive for the application at hand. There are instruments available in the market which could be modified to make moisture detection in ecc munitions simple, easy, rapid, and inexpensive.

\section{Background and Approach}

Moisture and its effects in the deterioration of the quality of materials and products have been extensively investigated. Moisture is classified as bound and free and can be specified by energetic, structural, or operational schemes, per Pyper et al. $(7,8)$. Methods for distinguishing between bound and free moisture are: (a) dynamic dielectric thermal analysis, (b) microwave attenuation analysis, (c) near infrared reflectance analysis, and (d) nuclear magnetic resonance spectroscopy (9). The classification of moisture as free or bound is not precise.

Application of an electric field to a material results in electric charge separation known as electric polarization. Polarization is classified as: (a) electronic polarization, caused by the displacement of electrons with respect to the nucleus, (b) ionic polarization caused by the displacement of negative ions with respect to positive ions, (c) dipolar or orientational polarization, which takes place in materials, known as polar, that have permanent dipole moments, (d) interface polarization, which takes place in electrically heterogeneous materials and is caused by the charging of the interfaces within the material; thus it depends on the dielectric properties of the constituent materials, and (e) counterion diffusion polarization, caused by ionic diffusion in electrical double layers adjacent to charged surfaces; counterion polarization effects are surface phenomena.

The dielectric permitivity $\epsilon$, and conductivity $\sigma$ of a material are macroscopic quantities by which the electrical properties of materials are specified. These quantities depend on the frequency $\omega=2 \pi \mathrm{f}$ of the applied field and are typically presented as

$$
\epsilon=\epsilon^{\prime}-j \sigma / \omega \epsilon_{0} \equiv \epsilon^{\prime}-j \epsilon^{\prime \prime}
$$


$\epsilon_{\mathrm{o}}=8.854 \times 10^{-12} \mathrm{~F} / \mathrm{m}$ is the permitivity of free space. $\epsilon^{\prime}$ represents the dielectric constant of the material and $\epsilon$ " represents the losses of the material. The losses of the material are typically expressed in terms of the loss tangent defined as

$$
\tan \delta=\epsilon^{\prime \prime} / \epsilon^{\prime}
$$

Water (moisture) is a polar material which can exist in a material in the forms of bound, free, or water that has chemically reacted with the host material to produce a new substance. The detection of moisture in a material, via dielectric measurements, relies upon the difference of the dielectric properties of dry and wet material, induced by the presence of highly polarizable water molecules. Water detection in a material dictates that a measurement $M$ can be expressed as a sum of

$$
M(f)=M_{m}(f)+M_{f}(f)+M_{b}(f)
$$

$M_{m}(f), M_{r}(f)$, and $M_{b}(f)$ are the dry material, free water, and bound water contributions, respectively; The chemically reacted water is neglected, if present it may change the properties of the material.

Analytical expressions of the dielectric permitivities of free and bound water have been developed and they are:

$$
\begin{array}{rlrl}
\epsilon_{f}^{\prime}(\omega) & =5+\frac{75}{1+\left(6.3 \times 10^{-11} \omega\right)^{2}}, & \epsilon_{f}^{\prime \prime}(\omega) & =\frac{4.7 \times 10^{-9}}{1+\left(6.3 \times 10^{-11} \omega\right)^{2}} \\
\epsilon_{b}^{\prime}(\omega)=88+\frac{.9}{1+\left(10^{-8} \omega\right)^{2}}, & \epsilon_{b}^{\prime \prime}(\omega)=\frac{9.0 \times 10^{-9} \omega}{1+\left(10^{-8} \omega\right)^{2}}
\end{array}
$$

The relative contributions of free and bound water to $\epsilon$ vary with frequency as follows (7),

$$
\begin{array}{cc}
\epsilon^{\prime \prime} / \epsilon^{\prime} \approx \text { constant } / \omega & \omega \leq 10 M H z \\
\epsilon^{\prime \prime} / \epsilon^{\prime} \approx \epsilon_{m}^{\prime \prime} / \epsilon_{m}^{\prime}+\epsilon_{f}^{\prime \prime} / \epsilon_{f}^{\prime}+\epsilon_{b}^{\prime \prime} / \epsilon_{b}^{\prime} & 10 M H z \leq \omega \leq 1 G H z \\
\epsilon^{\prime \prime} / \epsilon^{\prime} \approx \epsilon_{f}^{\prime \prime} / \epsilon_{f}^{\prime} & \omega \geq 1 G H z .
\end{array}
$$

These results are suitable for the evaluation of the dielectric losses (see Equation 8, later).

The experimental investigation in this project consisted of measurements of the variations of the capacitance and dissipation factor of ece coupons and simulated rounds as a function of moisture content. 


\section{Experimental}

\section{Equipment and Materials}

\section{Instruments}

The following instruments were used in the measurement of the electrical parameters of the cartridge simulators.

I\#1. Hewlett Packard 4192A LF Impedance Analyzer. This is a main frame laboratory instrument suitable for different types of electrical network parameter measurements. This instrument was used to measure capacitances, dissipation factors and impedance as a function of frequency of the simulations to determine the optimum frequency for moisture detection.

I\#2. B+K Dynascan corp. 830 Automatic Capacitance Meter. This is a hand-held battery operating instrument suitable for field type measurements. There are several low cost (about $\$ 100.00$ ) instruments of this type in the market.

I\#3. B+K Precision 875A LCR Meter. This is a hand-held battery operated meter suitable for field measurements. Its cost is $\$ 195.00$, and measures electrical inductance, capacitance, resistance, and dissipation factor.

I\#4. TRAMEX Moisture Encounter Model MF-1. This hand-held battery operating instrument provides non-destructive and non-invasive moisture detection in high electrical resistance materials such as wood, felt-roofing, plaster and brick. Appendix $A$ is the operating manual of the instrument. Its cost is $\$ 325.00$. The instrument detects moisture via measurement of the impedance of the material under test. The frequency of operation was measured to be about 12.5 $\mathrm{kHz}$. Two flat rubber electrodes provide electrical connection between the instrument and the subject under test. Electrical connection is made by holding the instrument so that the rubber electrodes are in firm contact with the subject under test. Note that this instrument will provide a reading without physical contact because of capacitive coupling. Three different scales provide quantitative measurement of moisture content in timber and wood products, felt roofing, and plaster or brick. The instruction sheet for this instrument is shown in the Appendix.

\section{Materials}

Five ccc coupons (about $9.5 \times 2.5 \times 0.4 \mathrm{~cm}$ ) left from the previous hardness and tensile strength test study were first chosen for moisture detection experiments, and instrument I\#1 was used for those moisture content measurements.

Three simulated cartridge cases (inert cases) were provided by the Future Ammunition Rearm System group for moisture detection experiments. The shape and configuration 
of each case was different. Their material composition was different from real cases in that nitrocellulose was not present. The three cases are identified as $\mathrm{C} \# 1, \mathrm{C} \# 2$, and

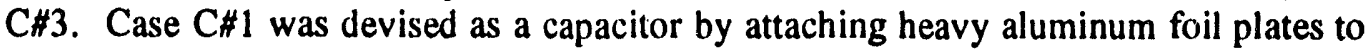
its external walls. Electrical leads from these plates were provided for measurement of capacitance and dissipation factor or equivalent impedances with instruments I\#1, $I \# 2$, or I\#3. Cases $C \# 2$ and $C \# 3$ were used without modification for measurement of moisture with instrument $\mathrm{I} \# 4$.

\section{Measurements}

Measurements were conducted on both coupons and simulated cartridge cases (inert cartridge cases).

a. Coupon measurements. Coupons were sandwiched between copper plates to simulate parallel plate capacitors. The assemblies or devices were held together by insulated metallic binding clips. The objective of the procedure was to provide non-destructive and non-invasive tests. A total of five devices ("devices \#1 - \#5") were constructed and measured using the Hewlett Packard 4192A LF Impedance Analyzer (I\#1). The frequency range of measurement was $1 \mathrm{KHz}$-to-13MHz. The capacitance $C$ and dissipation factor $D=\omega C R \approx \omega \epsilon_{0} \epsilon^{\prime} / \sigma$ were measured as a function of frequency.

b. Soft Wood Measurements. A crude capacitor simulation of the cartridge was devised as follows. Soft wood was used as the core material, a $2000 \mathrm{~mm}$ Pyrex beaker was used as a shell, and heavy aluminum foil was attached on the external walls of the beaker to simulate the metal plates of the capacitor. The premise was that the soft wood will absorb and desorb moisture in a manner similar to the real cartridge. Capacitance measurements were made on the Hewlett Packard 4192A LF Impedance Analyzer. The wooden cylinders, $124 \mathrm{~mm}$ diameter $\times 180 \mathrm{~mm}$ high, were fabricated from kiln-dried yellow pine, and were designed so they could be easily inserted and removed from the beaker. This wood has a typical specific gravity of 0.53 and a moisture content of $13.8 \%$ (10). The moisture content of wood changes with atmospheric conditions. For this experiment, the wooden cylinders were subsequently exposed to dry air and then room air $(\mathrm{RH} \approx 50 \%)$. The objective was to demonstrate the feasibility of detection of the change of the moisture content of wood via measurements of the change of the capacitance of the configuration.

c. Simulated Cartridge Case Measurements. Several experiments were made on the

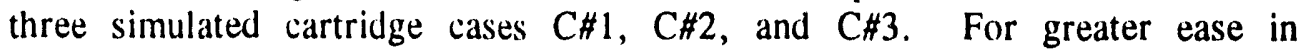
explanation, the experimental parts of these cartridge measurements are included in the results and discussion section. 


\section{Results and Discussion}

\section{Coupon Study}

Dry Measurements

The capacitance of all devices monotonically decreased with frequency. The dissipation factor measurements exhibited nonuniform variations, including the phenomenon of hysteresis with frequency. Because of time limitations and the crudeness of the experiment, no attempt was made at this stage to explain these variations on a physical basis.

\section{Wet Measurements}

The variations of the capacitance (C) and dissipation factor (D) with moisture are demonstrated with results from device \#5. C and D were measured with the device dry, and the results are shown in Figures 18 and 19. Then the device was immersed in water for about $20 \mathrm{~h}$. Starting an $\mathrm{h}$ after removal from the water, measurements of $C$ and $D$ were made every $h$ for $8 \mathrm{~h}$ at the frequency $f=1 \mathrm{MHz}$, with results shown in Figure 20. Seven hours after removal from the water, measurement of $C$ and $D$ were made as function of frequency, and the results are shown in Figures 18 and 19, along with the dry measurements. Peculiarities were noticed in the frequency range between $1 \mathrm{kHz}$ and $10 \mathrm{kHz}$; these results are shown in an expanded scale in Figure 21.

It is seen that both $C$ and $D$ increase with moisture, and that the changes are largest at low frequencies. The maximum change in $\mathrm{C}$ was $775 \%$ at $\mathrm{f}=1 \mathrm{kHz}$, and the minimum was $9.3 \%$ at $f=13 \mathrm{MHz}$. The changes in $\mathrm{D}$ were much larger: a maximum of $3250 \%$ at $\mathrm{f}=5 \mathrm{kHz}$, and a minimum of $95 \%$ at $\mathrm{f}=13 \mathrm{MHz}$. In addition, there is a "peak" in the graph of D, Figure 21. Such "peaks" are attributed to the presence of bound moisture in the material (11). More measurements of $C$ and $D$ were taken later, which demonstrated that the device recovered to its dry state (see Figure 20).

No attempt was made at this time to provide any physical explanation of these results. However, the results appear to have similar variations with frequency with those reported for moisture in triaminotrinitrobenzene (TATB) (7), Figure 22, obtained from measurements of the dielectric constant as a function of frequency. 


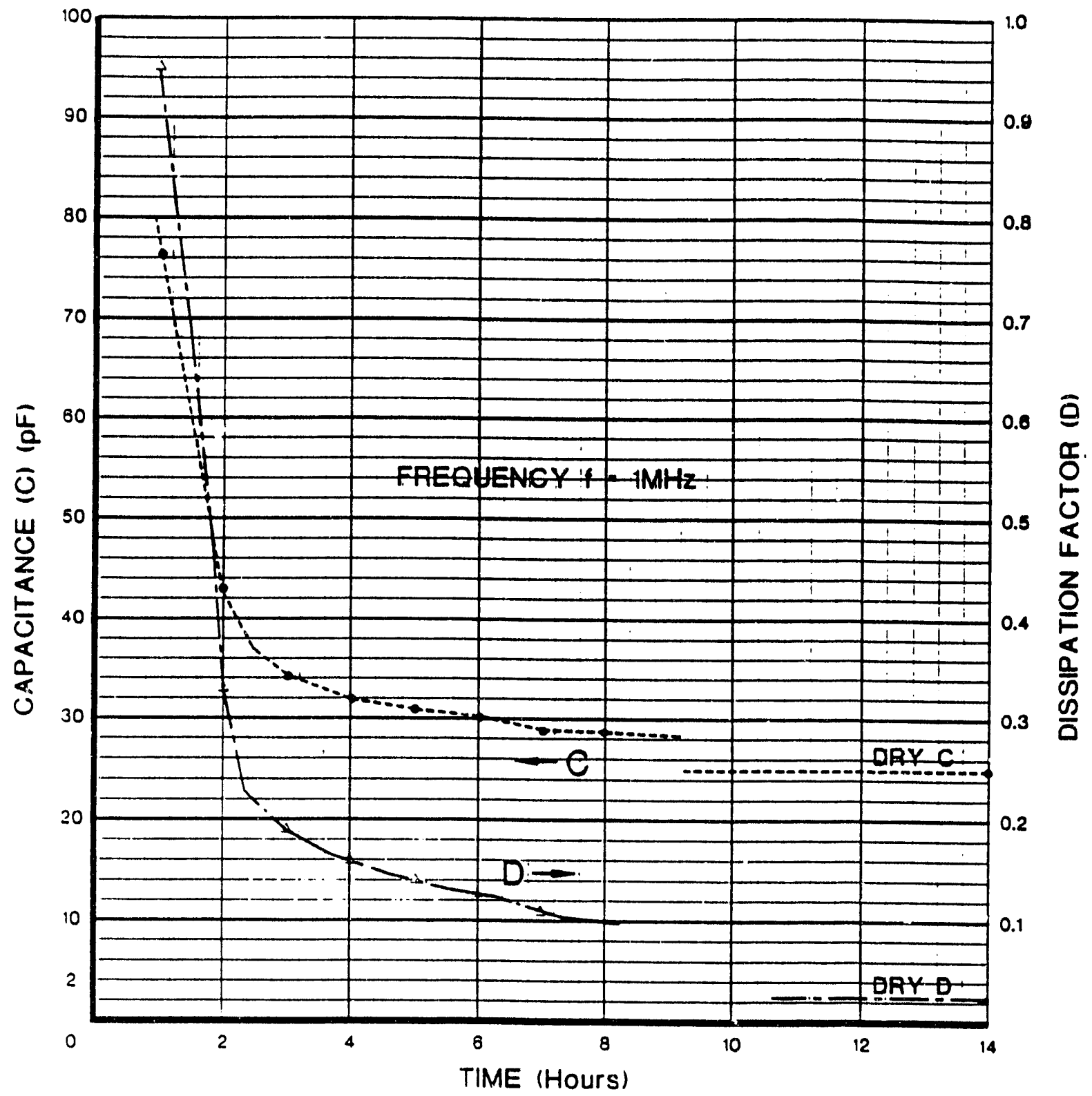

FIG. 18 CHANGE OF CAPACITANCE (C) AND DISSIPATION FACTOR

(D) OF DEVICE 5 AS A FUNCTION OF TIME, AFTER REMOVAL FROM THE WATER BATH, AT $t=0$ 


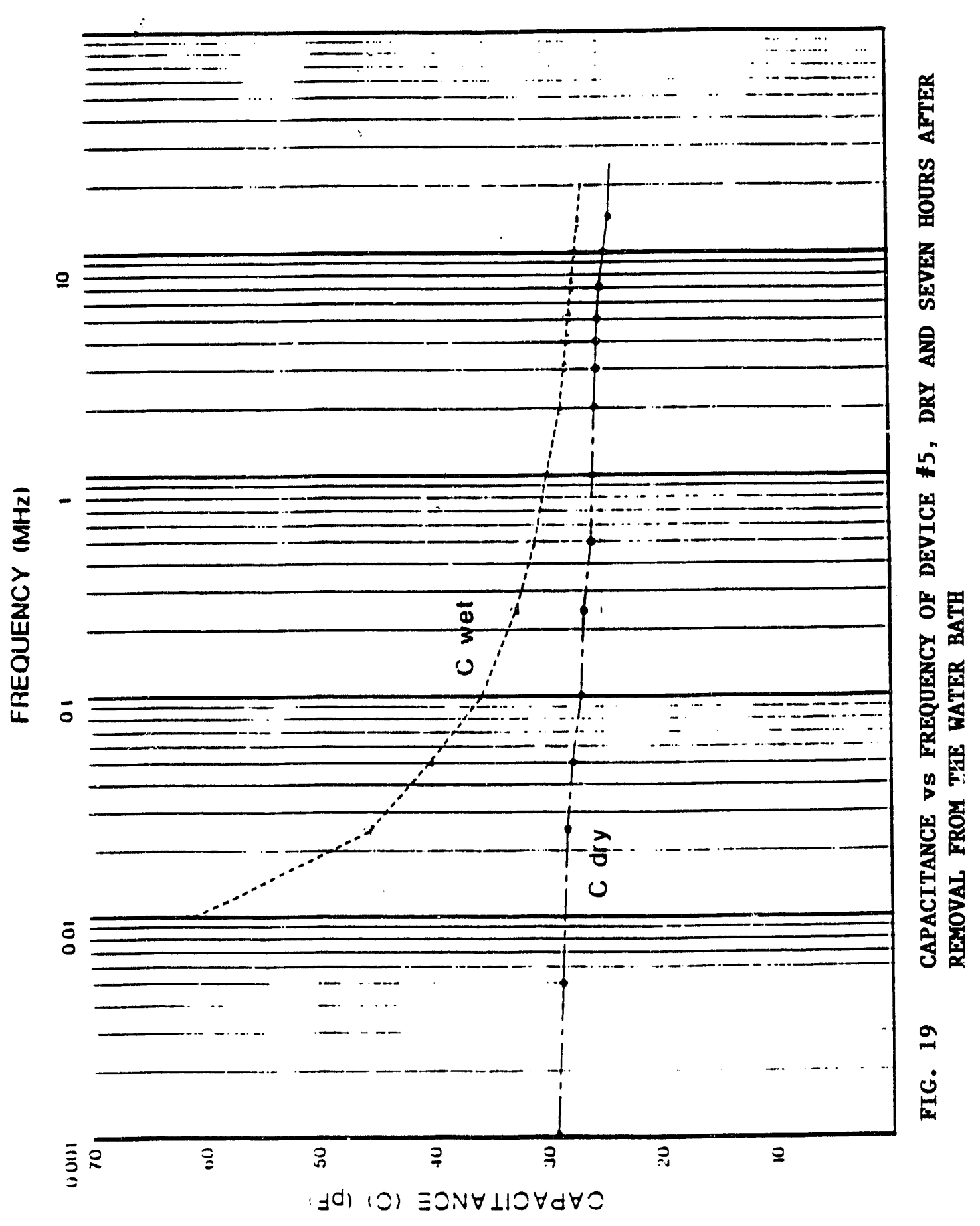




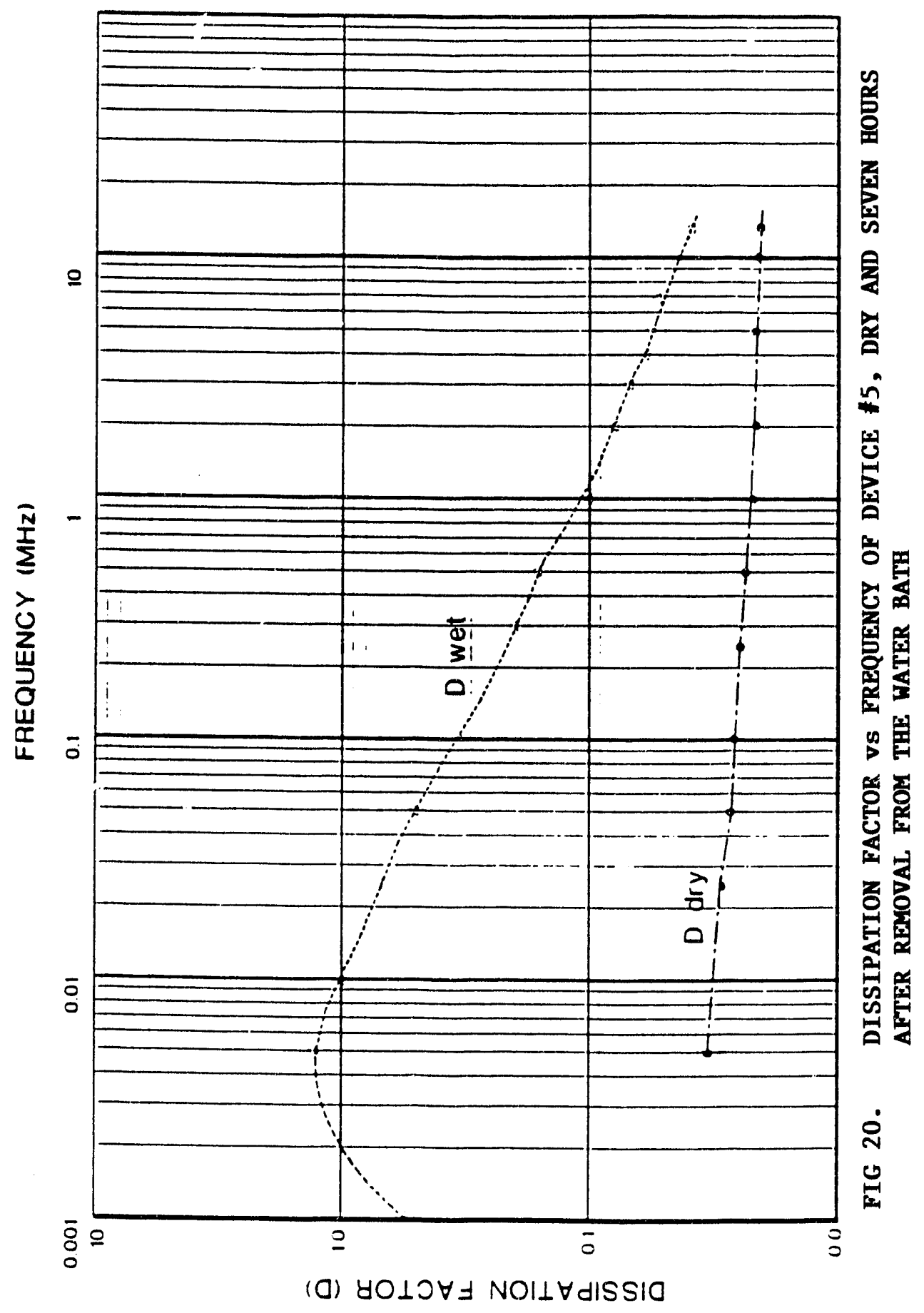


(a) $\forall O \perp O \forall \exists N O I \perp \forall d I S S I O$

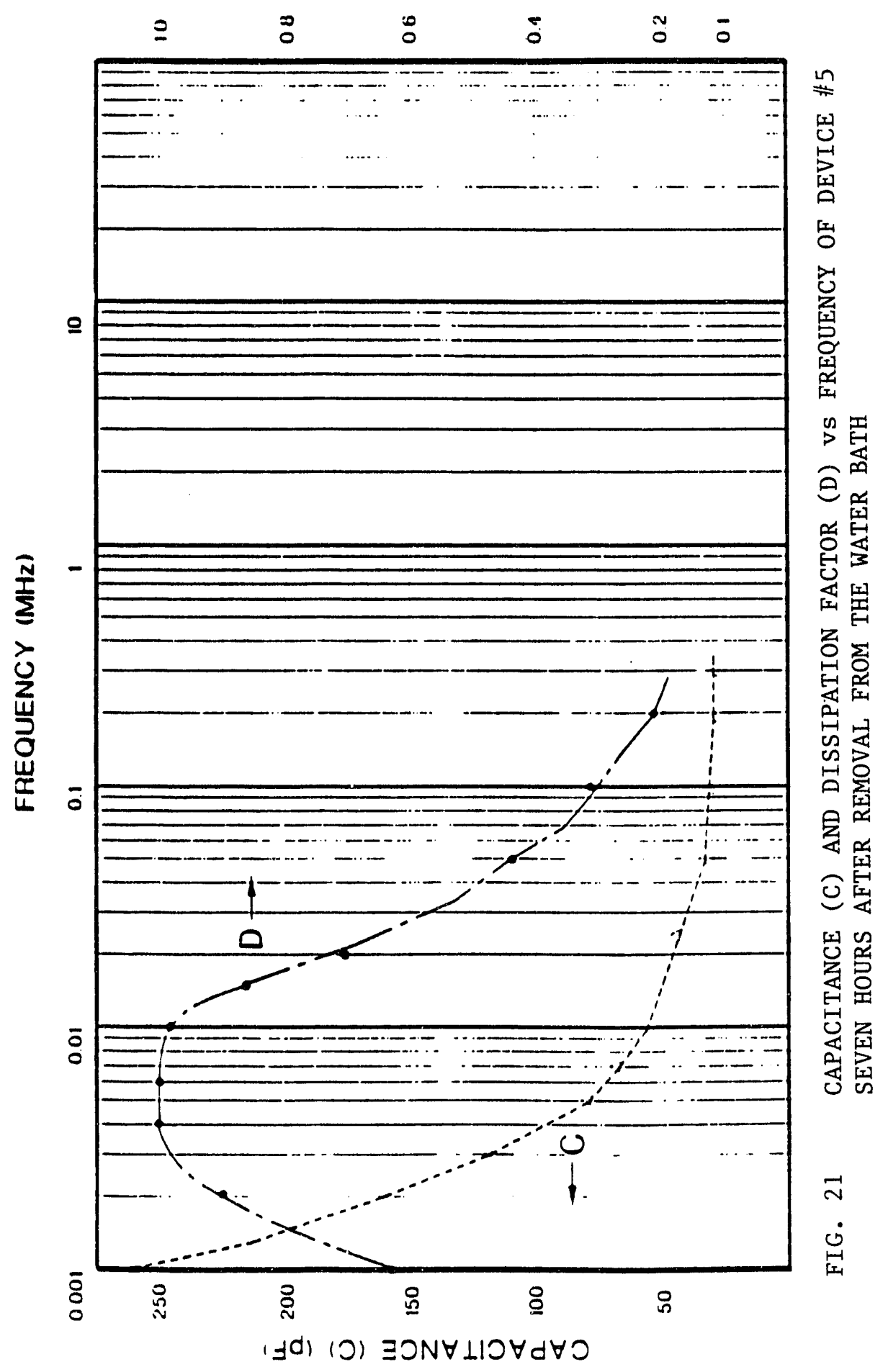




\section{Soft Wood Study}

The weight of the cylinder at room air was $1149 \mathrm{~g}$, and the water content (13.8\%) was 158.56 g. After several days in dry air the weight of the cylinder was $1128 \mathrm{~g}$, and the water content was about $137.6 \mathrm{gm}$, a reduction of about $13 \%$. The capacitance measurements were made at the frequency $f=4 \mathrm{MHz}$. The capacitance of the empty beaker was $15 \mathrm{pF}$ (picofarads). The capacitance with the dry cylinder inserted was $20.45 \mathrm{pF}$. After the cylinder was exposed to room air for about $24 \mathrm{~h}$, the capacitance was $21.6 \mathrm{pF}$. Thus, the capacitance change of the whole configuration due to room air moisture was $1.15 \mathrm{pF}$ or $5.6 \%$. This is smaller than the $13 \%$ change in moisture obtained from weight measurements. This difference can be explained as follows. The total capacitance $C$ of the simulator consists of the series connection of the capacitance of the glass walls $C_{b}$, the capacitance of the air layer between the glass and the wood $C_{a}$, and the capacitance of the wood $C_{w}$. Thus, the capacitance of the simulator $C$ is

$$
\frac{1}{C}=\frac{1}{C_{g}}+\frac{1}{C_{a}}+\frac{1}{C_{w}}
$$

Taking the differential of this equation, the expression of the relative change of $C_{w}$, in terms of the relative change of the measured capacitance $C$, is

$$
\frac{\Delta C_{w}}{C_{w}}=\left(1+\frac{C_{w}}{C_{a}}+\frac{C_{w}}{C_{g}}\right) \frac{\Delta C}{C}
$$

It is seen that the relative change of $C_{w}$ is larger than the relative change of the measured capacitance $\mathrm{C}$, and depends on the values of the capacitances of the simulator. The dissipation factor of the simulator was not measured in this experiment. 


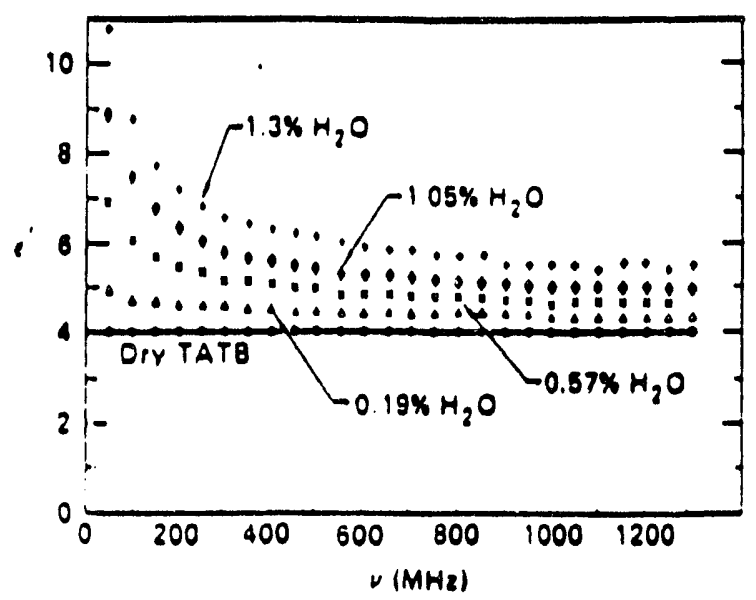

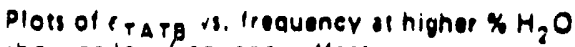
inowing low liogueney ofleet

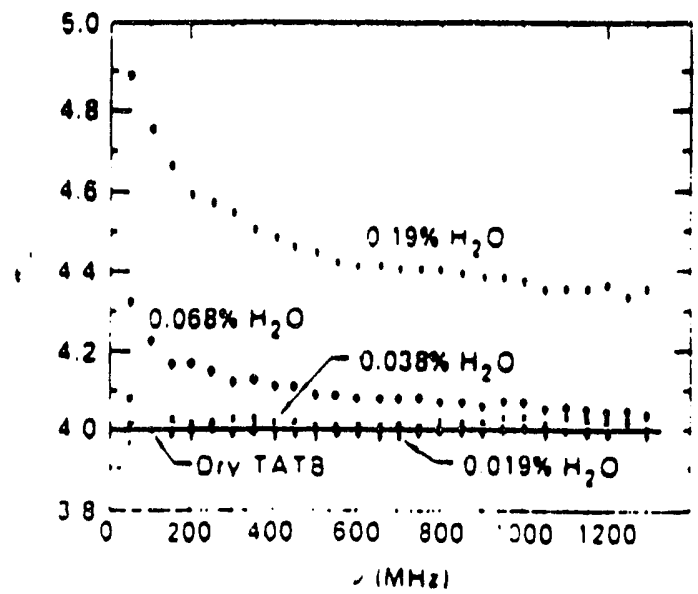

Plot showing detection limit of $0.04 \times \mathrm{H}_{2} \mathrm{O}$ in TATB

Figure 22. Dielectric constant vs frequency. Source: Pyper (7). 


\section{Simulated Cartridge Study}

\section{A. C\#2 Measurements}

Case C\#2 data: height $48.5 \mathrm{~cm}$, diameter $15 \mathrm{~cm}$, mass (dry) $679.5 \mathrm{~g}$. Moisture content (dry) reading $\approx 10 \%$ (bottom of scale). All moisture measurements on $\mathrm{C} \# 2$ were made using the Tramex moisture encounter ( $(\# 4)$ on Scale \#1. Visual inspection revealed that $\mathrm{C} \# 2$ is made up of two visually different sections glued together. No information is available at this time on the material composition of $\mathrm{C} \# 2$, but we believe that they are wood fiber analogs of the wood fiber/nitrocellulose used in actual ccc munitions.

$\mathrm{C} \# 2$ was filled with water for about $24 \mathrm{~h}$. Then the water was removed and the moisture content was evaluated by weight measurements. The maximum amount of water absorbed was $75.3 \mathrm{~g}$. or $\approx 11.1 \%$ of the mass of $\mathrm{C} \# 2$. Subsequently, $\mathrm{C} \# 2$ was exposed to the ambient room environment to dry for several days. During this time the moisture content of $\mathrm{C} \# 2$ was recorded from measurements by weight and by $\mathrm{I} \# 4$. The results are depicted in Figure 23. The plot indicates that the moisture content of the two section of $\mathrm{C} \# 2$, as recorded by $\mathrm{I} \# 4$, are not the same. In addition, readings of $\mathrm{I} \# 4$ varied along the height and the periphery of both sections. The latter indicates that water is not uniformly absorbed in the walls of $\mathrm{C} \# 2$, therefore, the composition of $\mathrm{C} \# 2$ probably is not uniform. This could be due to an uneven distribution of additive in the case wall.

Because the rubber electrodes of I $\$ 4$ are configured in a flat plane and the surface of $\mathrm{C} \# 2$ is cylindrical, complete contact of the electrodes with the surface of $\mathrm{C} \# 2$ during the measurements was impossible. In fact, even partial contact was possible only with I $\# 4$ oriented along the height of $\mathrm{C} \# 2$. Thus, no quantitative absolute meaning can be attached to the moisture readings on the scale of the I\#4.

\section{B. CH1 Measurements}

Four sets of experiments were conducted using $\mathrm{CH1}$.

1. C\#1 Tests of Different Meters. C\#1 data: height $46.5 \mathrm{~cm}$, diameter $15 \mathrm{~cm}$, mass (dry, including the attached electrodes) $599.6 \mathrm{~g}$. The C\#1 configuration was assembled for measurements with instruments $1 \# 1$, $I \# 2$, and $I \# 3$. All these instruments are designed to measure the capacitance of electronic components, which are typically low loss and reasonably linear, and frequency independent devices. The capacitance of the configurations of $\mathrm{C} \# 1$ described below varied with frequency and, thus, was not linear. It was noticed that the capacitance readings of the three instruments were substantially different. Eventually, instruments I\#2 and I\#3 stopped functioning all together. It was concluder, therefore, that instruments $I \$ 2$ and $I \# 3$ are not suitable for measurement of capacitance, as a means of moisture detection, without major moditications. 


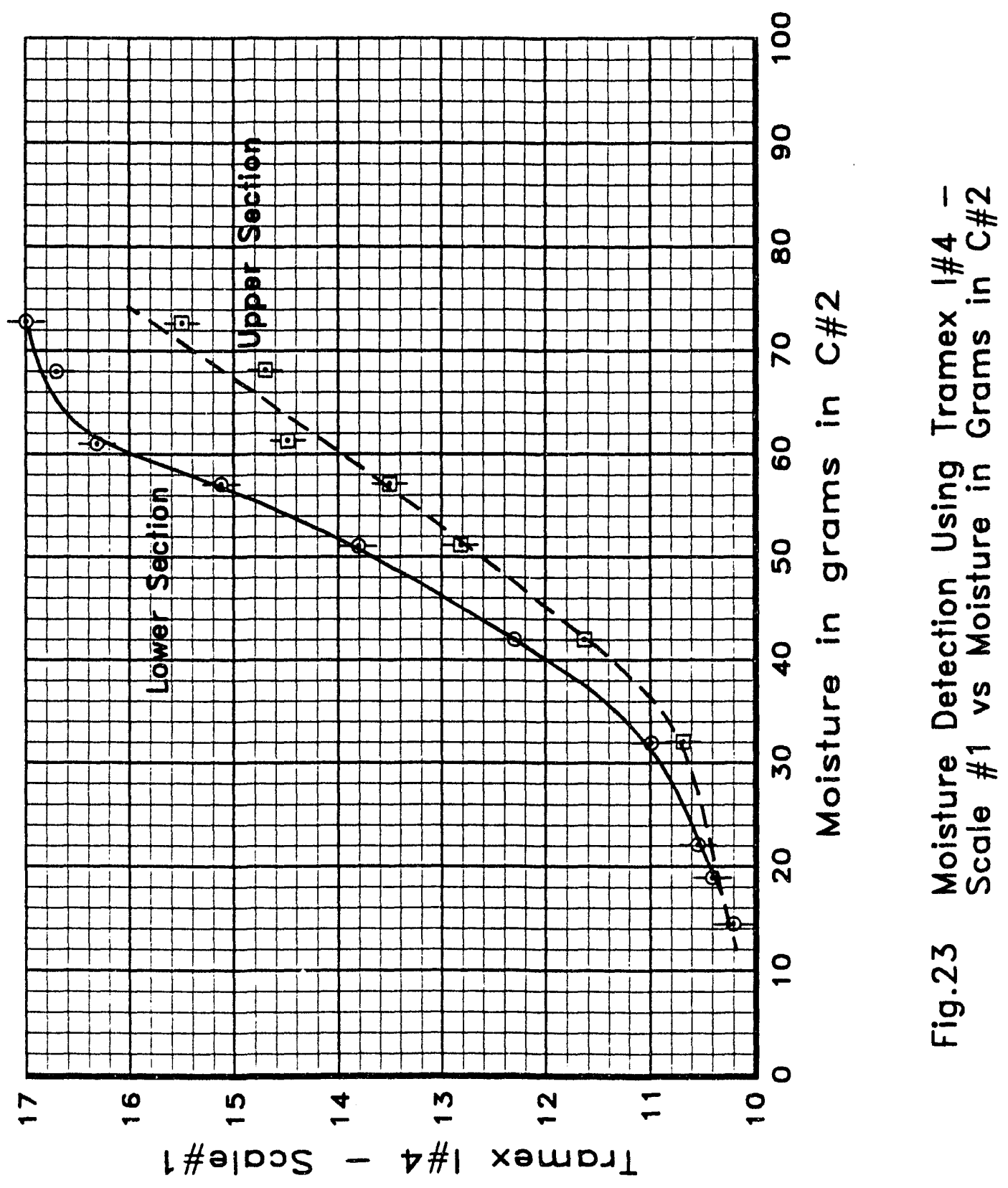


2. $\mathrm{C} \# 1$ and Soft Wood. The variations of the capacitance $C$ and dissipation factor $D$ with frequency of $\mathrm{C} \# 1$ empty, were measured using $\mathrm{I} \# 1$. The results are plotted in Figure 24. Then the soft wood (dry) cylinder was inserted in the center of C\#1, and the $\mathrm{C}$ and $\mathrm{D}$ of this assembly was measured. The results are plotted in Figure 25. As seen from Figures 24 and 25 , there are only slight changes in C and D due to the insertion of the wooden cylinder. Subsequently, the wooden cylinder was immersed in water for several days. The weight of the wooden cylinder was 1392 $\mathrm{g}$ dry, and $1751 \mathrm{~g}$ wet, thus, the water absorbed was $359 \mathrm{~g}$. The wet cylinder was reinserted in $\mathrm{C} \# 1$ and allowed to dry in the room environment for several $d$. During this time $\mathrm{C}$ and $\mathrm{D}$ of the assembly were measured with $\mathrm{I} \# 1$ at the frequencies of $1 \mathrm{kHz}, 10 \mathrm{kHz}, 100 \mathrm{kHz}, 1 \mathrm{MHz}$, and $10 \mathrm{MHz}$; the results for $\mathrm{f}=$ $1 \mathrm{kHz}, 10 \mathrm{kHz}$, and $100 \mathrm{kHz}$ are depicted in Figures 26 and 27 . The relative changes of $C$ and $D$ at $f=1 \mathrm{MHz}$ and $f=10 \mathrm{MHz}$ were too small to be significant.

It is seen from Figures 26 and 27 that the changes in C and D were larger at lower frequencies relative to the changes at higher frequencies. The initial values of $\mathrm{C}$ and $D$ were small then increased with time to peak values at $5 \mathrm{~d}$ and eventually decayed to the dry condition values. The moisture was initially concentrated in the wooden cylinder. As the cylinder dried the moisture migrated to the walls of $\mathrm{C \# 1}$ where it was absorbed. Thus, during the early days of the experiment the moisture concentration in the cylinder was decreasing, while the moisture concentration in the case wall of $\mathrm{CH} 1$ was increasing. Eventually the moisture concentration of the whole assembly decreased to the dry levels. It appears therefore that the variations of $C$ and $D$ follow the moisture concentration in the walls of C\#1 rather than the moisture levels in the cylinder. This is in agreement with the results of Figures 24 and 25 , which indicate that the most significant contribution to $C$ and $D$ comes from $\mathrm{C} \# 1$ itself and not the material it contains. The measurements thus appear specific to the case wall.

3. C\#1 Filled with Sawdust. C\#1 was filled with sawdust and the weight changed from $599.6 \mathrm{~g}$ to $1897 \mathrm{~g}$. The moisture content of the sawdust was measured with I $\# 4$ (Scale\#1) to be about $10 \%$. C and D of the configuration was measured with I\#1 in the frequency range of $1 \mathrm{kHz}$ to $10 \mathrm{MHz}$. The results for the capacitance change are shown in Figure 28. Similar relative changes were found for the dissipation factor $D$. Comparison of the $C$ and $D$ values with the corresponding $C$ and $D$ values of the empty $C \# 1$ revealed that the changes were small. The latter supports the previous observations that the most significant contributions of $\mathrm{C}$ and D come from C\#1 itself. Subsequently the configuration was enclosed in a high moisture environment for several days in order to absorb moisture. The mass increased from $1897 \mathrm{~g}$ to $1912.3 \mathrm{~g}$ for a change $\mathrm{dW}=24.3 \mathrm{~g}$. Measurements of $C, D$, and $\mathrm{dW}$ were made for several days at the frequencies of $1 \mathrm{kHz}, 10 \mathrm{kHz}$, $100 \mathrm{kHz}, 1 \mathrm{MHz}$, and $10 \mathrm{MHz}$. The changes were small. For example, at $\mathrm{f}=$ $1 \mathrm{kHz}, \mathrm{C}$ changed from $59.6 \mathrm{pF}$ dry $(\mathrm{dW}=0)$ to $73.4 \mathrm{pF}$ for $\mathrm{dW}=24.3 \mathrm{~g}$, to $67 \mathrm{pF}$ for $\mathrm{dW}=16 \mathrm{~g}$. The drying process was very slow and the experiment was terminated at this point. 


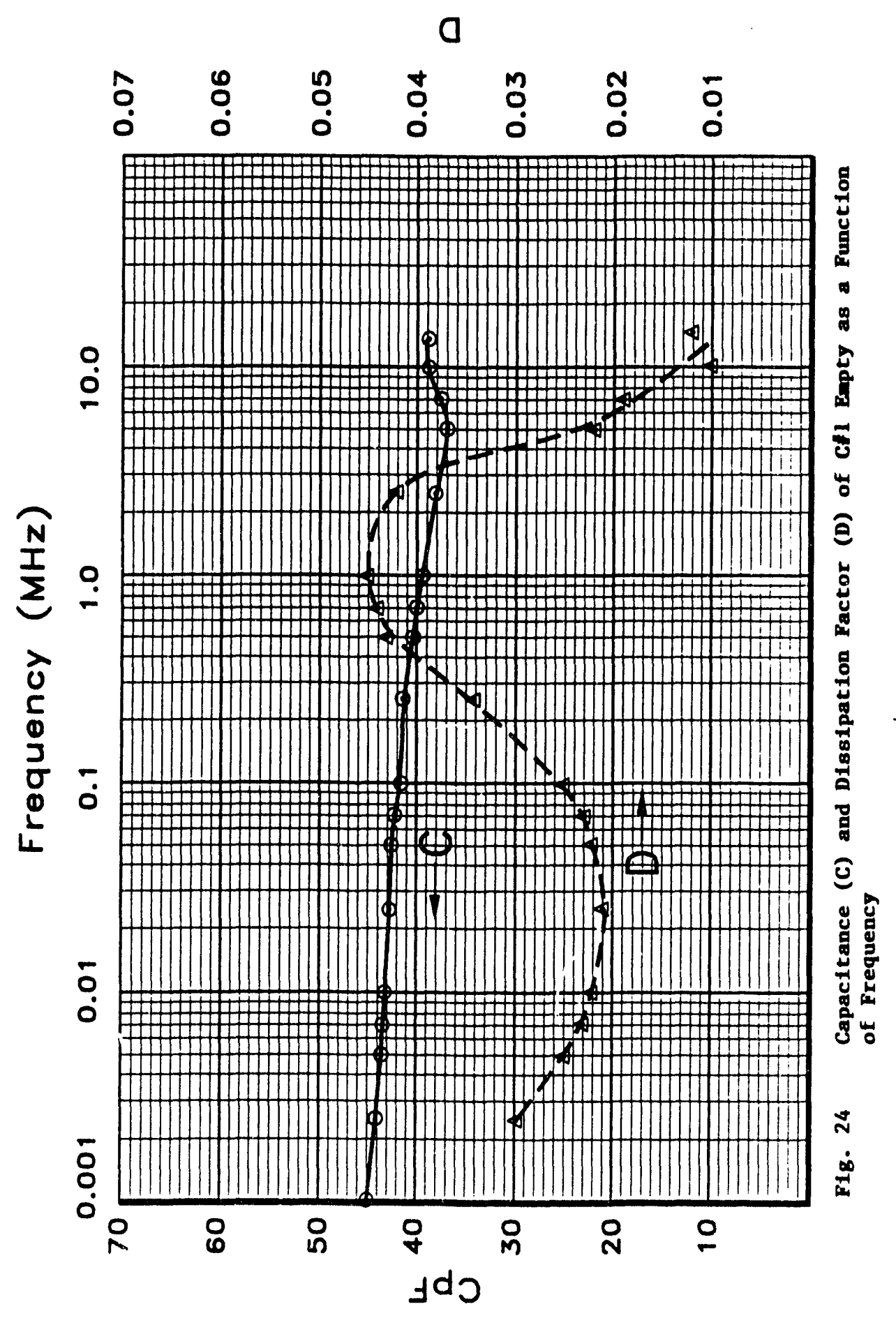




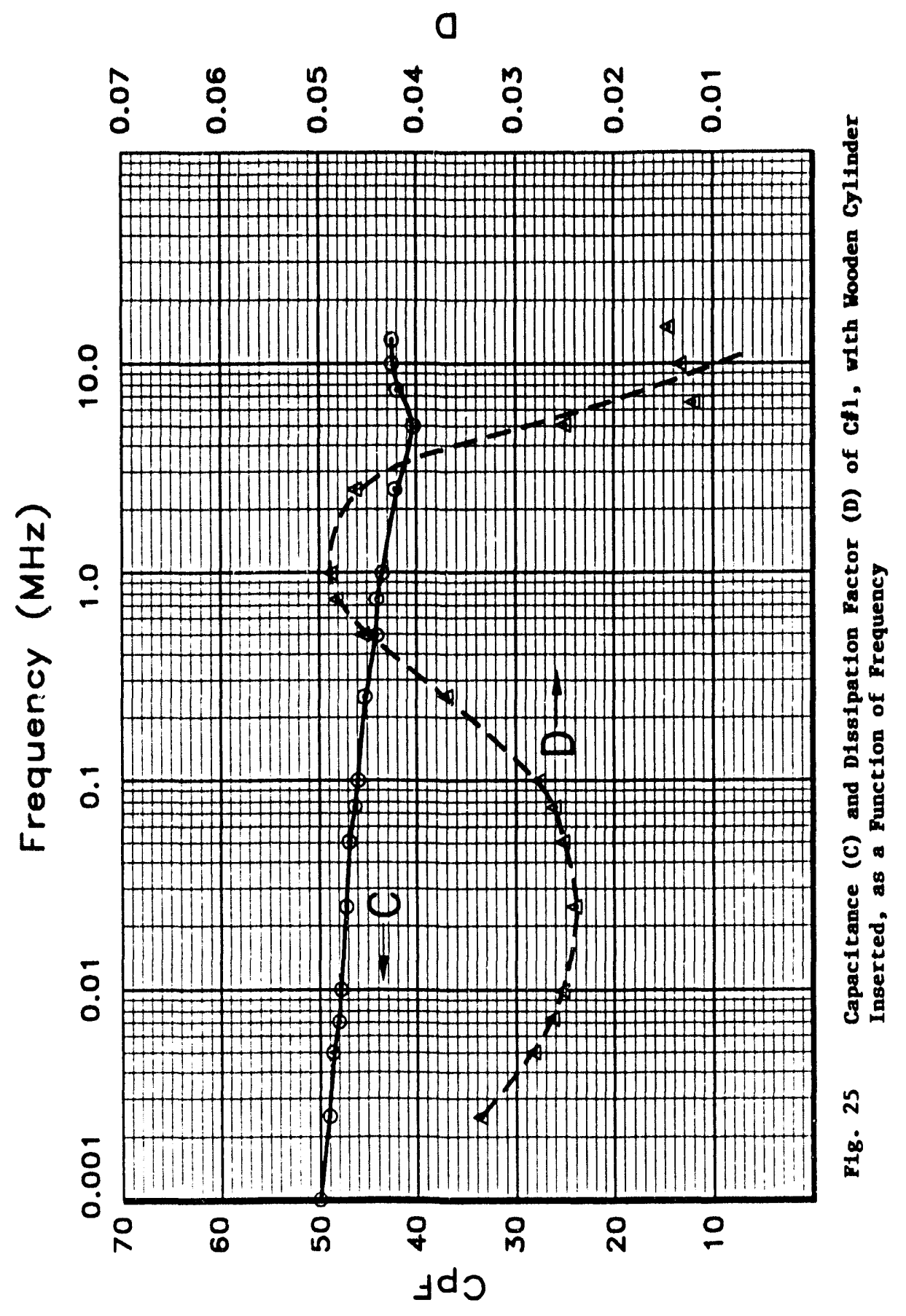




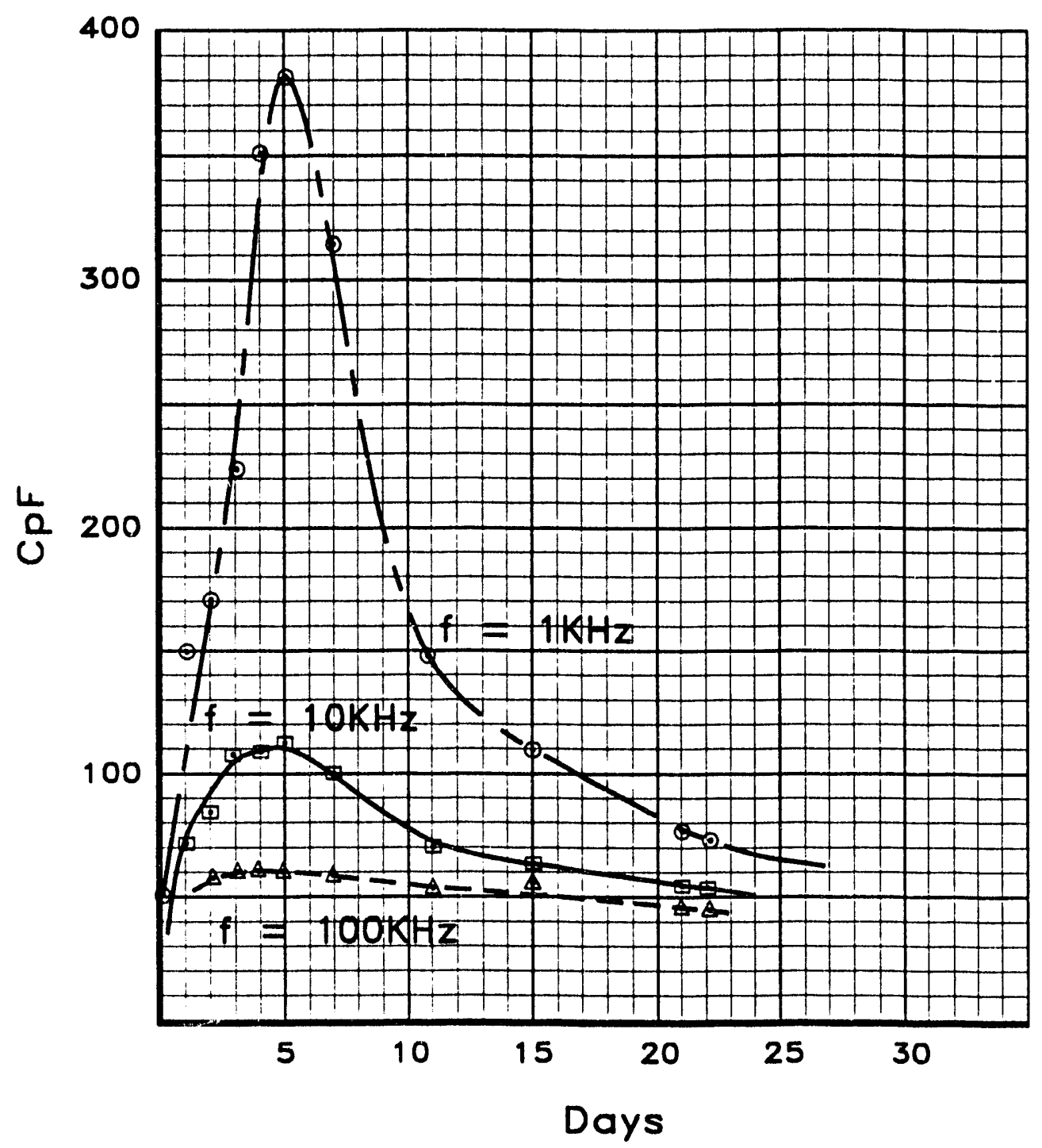

Pig. 26 Variation of Capacitance (C) vs Time of cfl with Wet Cylinder at its Center 


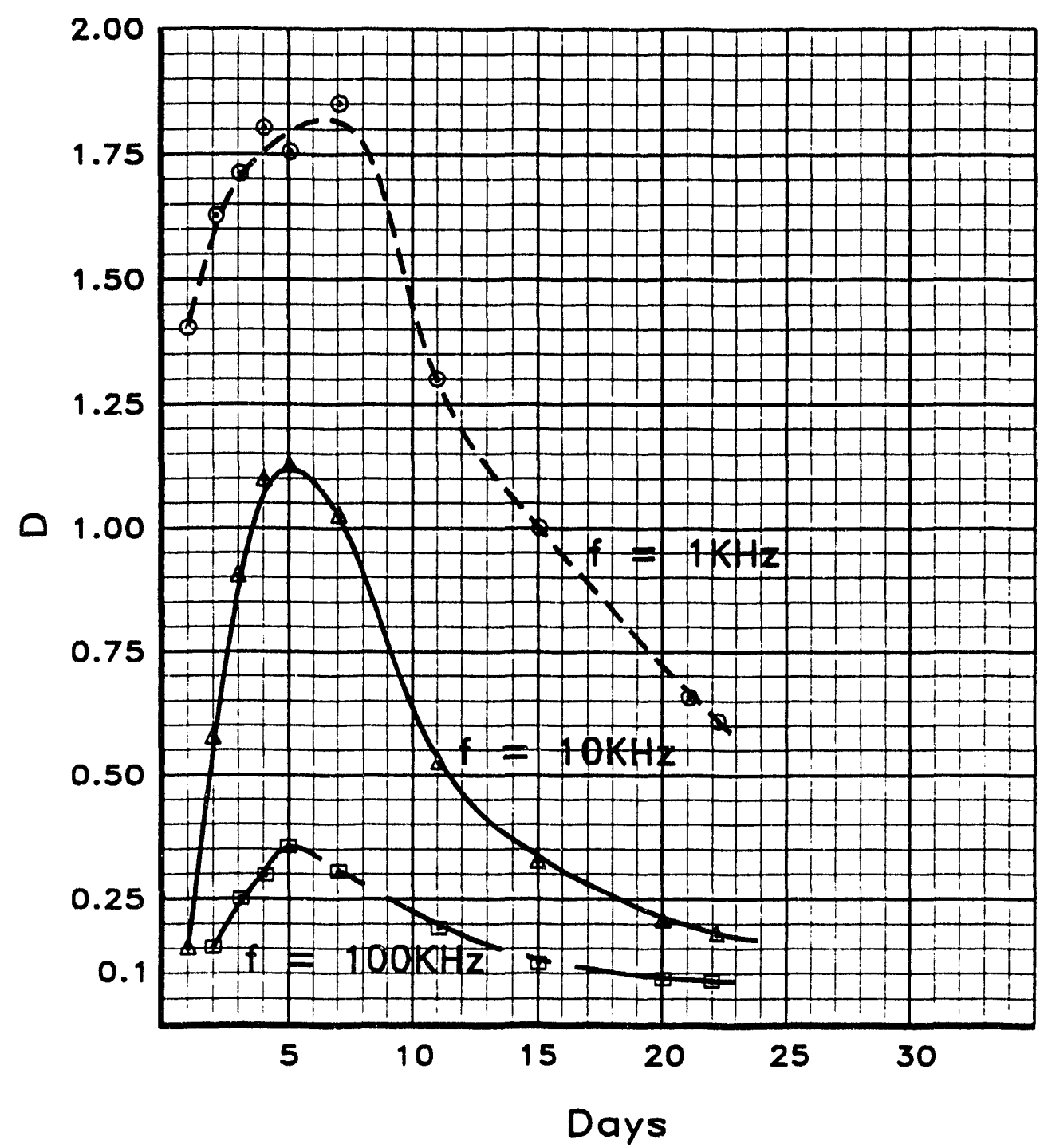

F1g. 27 Variation of (D) vs Tine of Cfl with Wet Wooden Cylinder at the Center 


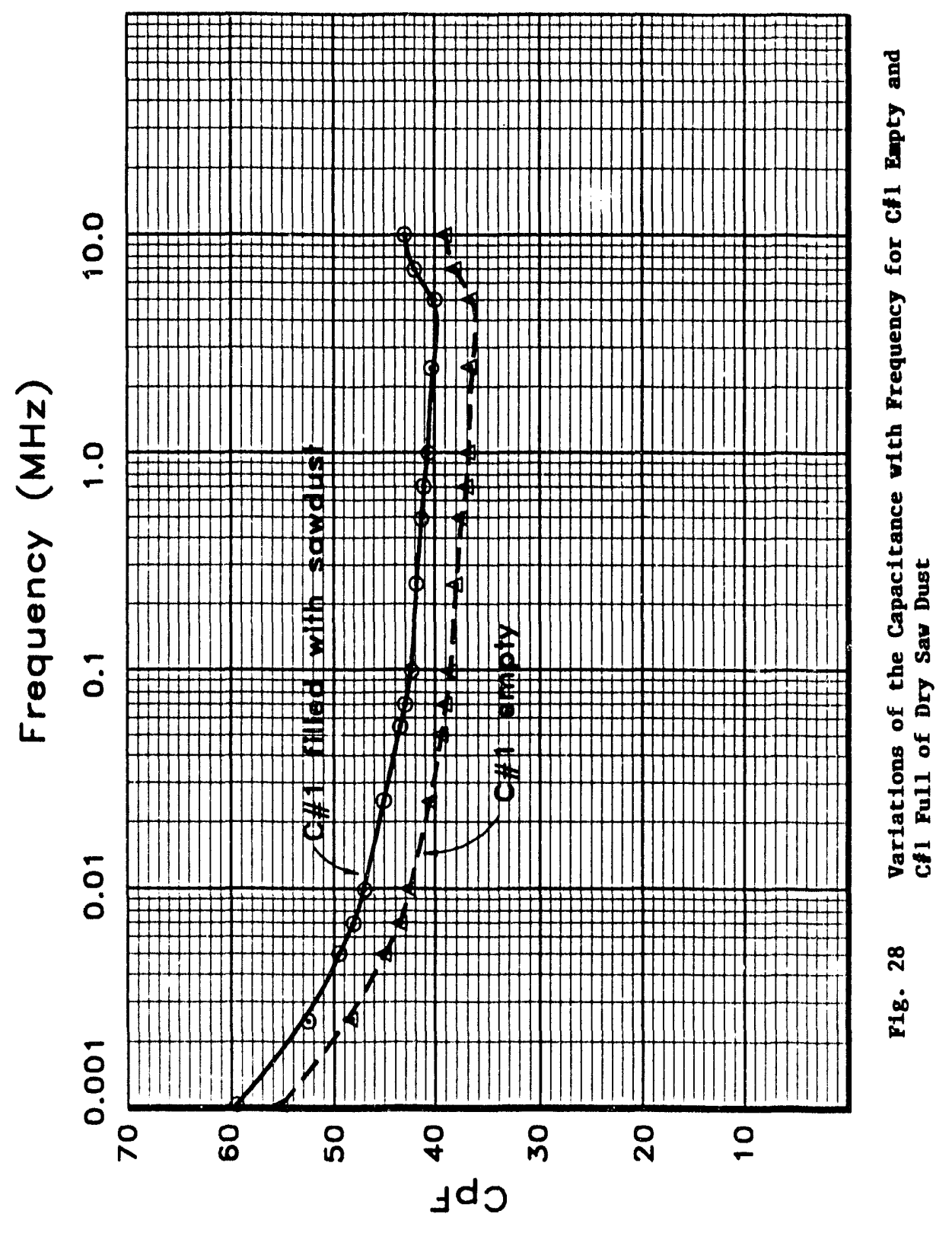


4. C\#I Filled with Wet Sawdust. Subsequently, C\#1 filled with sawdust was flooded with water and was allowed to dry. During the drying period the capacitance and impedance of the configuration along with the water content was measured. At the start of the measurements the mass of the configuration was $4807 \mathrm{~g}$. Thus, the excess water from the flooding was $\mathrm{dW}=2.91 \mathrm{~kg}$. The results of these measurements for the duration of ahout five weeks are shown in Figures 29 and 30.

Figure 29 depicts the change of capacitance as a function of excess water content at the frequencies of $1 \mathrm{kHz}, 10 \mathrm{kHz}, 100 \mathrm{kHz}$, and $1 \mathrm{MHz}$. These results show that the capacitance increases as the water content increases. The relative changes of the capacitance are larger at lower frequencies. For example, at $\mathrm{dW}=2.91 \mathrm{~kg}$, the ratios of the wet to dry capacitances $\left(C_{\text {wot }} / C_{\text {dry }}\right)$ were: 70000 at $1 \mathrm{kHz}, 7900$ at $10 \mathrm{kHz}, 810$ at $100 \mathrm{kHz}$, and 73 at $1 \mathrm{MHz}$. Similar measurements were made of the dissipation factor $D$. The $D$ measurements showed some erratic variations with water content.

Figure 30 shows the variation of the magnitude of the impedance of the configuration as a function of frequency, with water content as a parameter. These measurements indicate that the impedance variations with water content are more uniform with frequency than the capacitance variations (Figure 29). The latter results suggest that the frequency of operation of a moisture instrument based upon impedance may not be as restricted for capacitance measurements. 


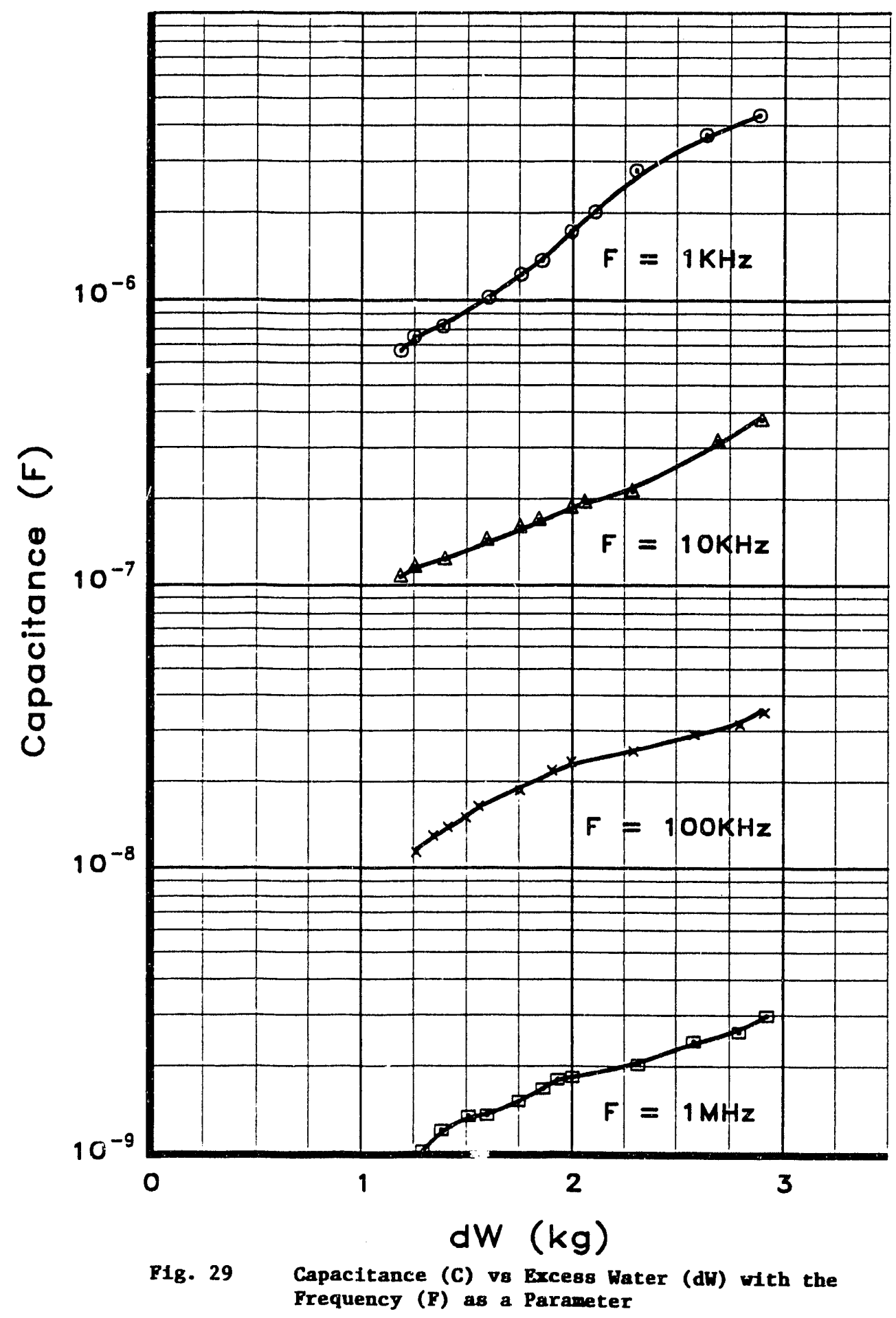




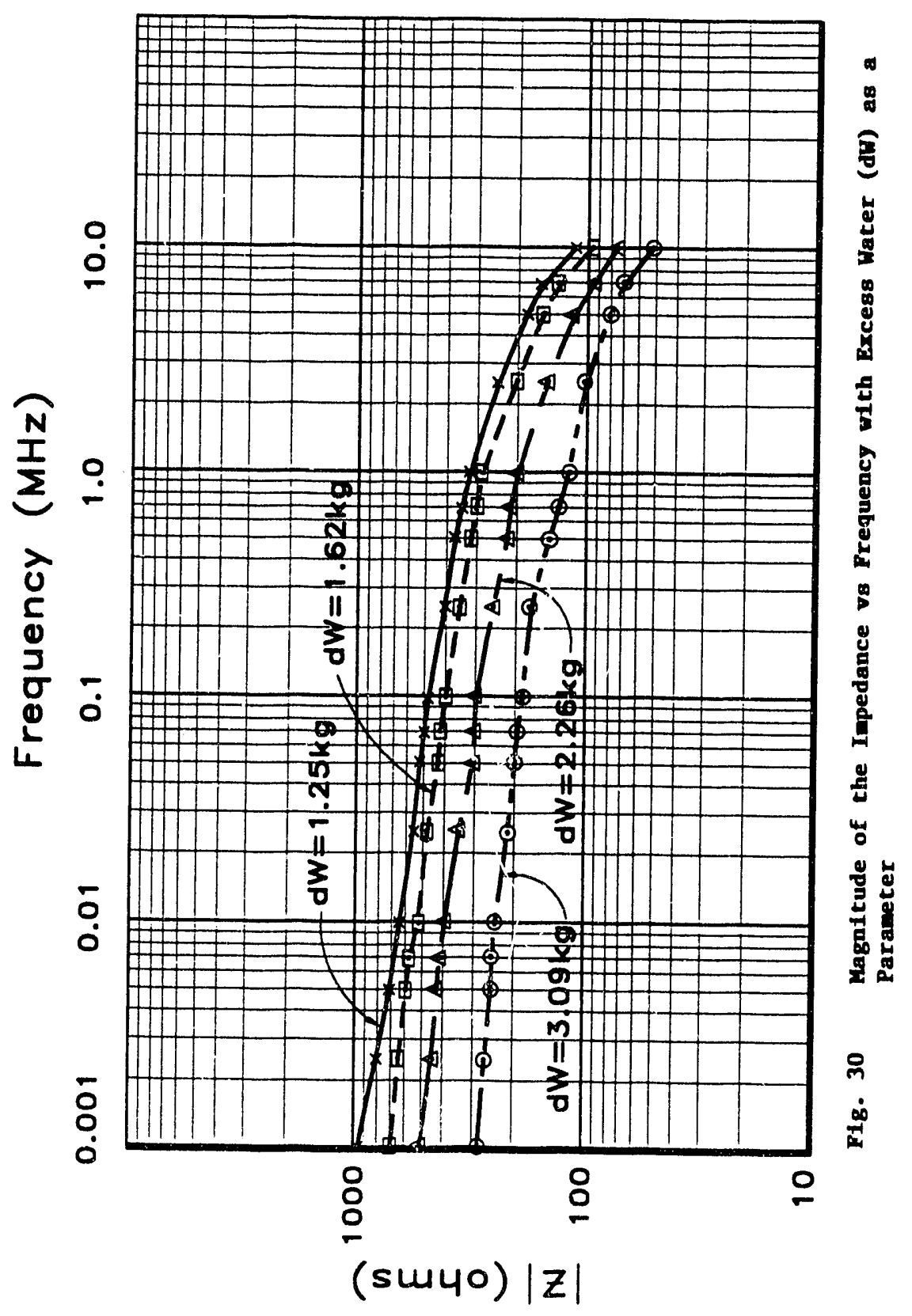




\section{C\#3 Measurements}

Two experiments were conducted with $\mathrm{C} \# 3$.

1. $\mathrm{C \# 3}$. C\#3 data: height $51 \mathrm{~cm}$, diameter $15 \mathrm{~cm}$, mass (dry) $743 \mathrm{~g}$. C\#3 was indented to be used for moisture measurements with instrument $1 \# 4$, Scale \#1. The moisture content of $\mathrm{C} \# 3$ dry was $\approx 10 \%$. C\#3 was filled with sawdust and the mass of the configuration increased to $2268 \mathrm{~g}$., and the moisture content was $\approx$ $10.2 \%$. Subsequently, the configuration was placed in a moist environment along with $\mathrm{C \# 1}$ (see above) for several days and its mass was changed to $2346.6 \mathrm{~g}$ and the $I \# 4$ reading increased to $\approx 13 \%$. In subsequent measurements the excess moisture was gradually reduced with time, but the $1 \# 4$ reading returned shortly to $\approx 10.2 \%$ (dry state). The experiment was abandoned at this stage.

2. C\#3 Filled with Wet Sawdust. Subsequently, the configuration of C\#3 filled with sawdust was flooded with water and was allowed to dry. During drying, the water content was recorded from mass measurements and moisture measurements with instrument $\mathrm{I} \# 4$. At the start of the measurements the excess water content due to flooding was $\mathrm{dW}=3.4 \mathrm{~kg}$, and the $\mathrm{I} \# 4$, Scale \#1 indication was about $19.5 \%$. These measurements were continued for about 5 weeks. During this time the excess water content fell to $\mathrm{dW}=1.82 \mathrm{~kg}$, while the $\mathrm{I} \# 4$, Scale \#1 indication remained nearly unchanged from the initial reading. These results suggest that the reading of I\#4 depends mainly on the moisture content of the case (surface of the configuration) which apparently has not changed.

\section{Conclusions and Recommendations}

The results of this study indicate that it is feasible to measure moisture in ccc munitions via measurements of their dielectric properties. There is substantial technology available for such measurements in the frequency range from $50 \mathrm{~Hz}$ to $1300 \mathrm{MHz}$. The results show that measurement of capacitance and dissipation factor at frequencies around $1 \mathrm{kHz}$ to $10 \mathrm{kHz}$ should be most suitable. However, if the problem is caused by moisture which has chemically reacted to change the dielectric properties of the materials, the above results may be of no value. Much more information is needed to arrive at specific recommendations.

What is envisioned is a hand-held battery-operated device which could be quickly held to the surface of the cartridge case and provide a reading of the moisture content. Small inexpensive capacitor meters of the type $I \$ 2$ and $I \# 3$ are commercially available and could probably be modified and adapted for this service. If a custom designed capacitance meter is needed, no extensive development is anticipated. This procedure has been apparently incorporated in the TRAMEX Moisture Encounter, I\#4. The I\#4 appears to be the most suitable instrument for moisture detection in ecc munitions because it is designed as a moisture detection instrument. 
The most obvious modification of $\mathrm{I} \# 4$ for use with $\mathrm{ccc}$ munitions is the change of the geometry of its electrodes, from plane to cylindrical, to fit firmly the surface of the cartridge case. Changing the frequency of operation from $12.5 \mathrm{kHz}$ to lower values may enhance the sensitivity of the instrument. An appropriate scale suitable for ccc must be developed. The size and shape of the electrodes should be re-evaluated to match the new application. In addition, modifications such as addition of conducting bands (strips) in the cartridge case itself may be considered for easier access.

It is emphasized that the reading of the instrument $1 \# 4$ depends strongly on the moisture content of the case wall (near the surface of munitions). Thus, the I\#4 is not suitable for measurement of the moisture of the bulk contents of the munitions.

It is feasible that similar electrical measurements could be used to detect the disbonding of skive joints in munitions. If this is successful, the detection of disbonding could be done by the addition of conductive rings (electrodes) on each side of the skive joint. Consideration of this investigation also is recommended.

A continued investigation is recommended, in which the TRAMEX Moisture Encounter is modified and optimized for the rapid determination of moisture in the case wall of ccc munitions. This effort would require both laboratory work at Oak Ridge and interaction with Picatinny Arsenal to test the device on actual munitions. The product of this work would be a prototype device with the modifications and a draft operating protocol.

It is also possible that such measurements could be built into automatic rearm systems to evaluate rounds (and trigger their rejection, if necessary) before they are loaded. That would require the development of a different device from the hand-held meter considered above, and a separate effort. 


\title{
PRELIMINARY EVALUATION OF INFRARED SPECTROSCOPY
}

\begin{abstract}
Introduction
Infrared spectroscopy is a powerful nondestructive analytical tool which can discern the chemical bonds in a material from its absorption of infrared radiation. To determine whether diffuse-reflectance Fourier transform infrared spectroscopy (DRIFT) measurements could be useful for determination of the moisture content in ccc material, ccc coupon samples were first dried, and then exposed to high humidity for varying periods of time. A series of DRIFT spectra were acquired as a function of exposure time to moisture.
\end{abstract}

\section{Experimental}

A 1" $\times$ 4" sample of ccc coupon was dried by storing in a desiccator with Drierite at room temperature for approximately $120 \mathrm{~h}$. Four smaller samples were then cut from this sample and weighed. One of these smaller samples (Sample 1) was placed back in the desiccator, while the other three (Samples 2-4) were placed in a closed chamber containing an open $40 \mathrm{~mL}$ vial of distilled, deionized water. After 24 additional h, the samples were weighed again. Sample 1 and Sample 2 were then cut apart to expose the cellulose nitrate interior, and DRIFT spectra were obtained of the cellulose nitrate faces of each these samples. The samples were returned to the desiccator (Samples 1 and 1a, resulting from cutting Sample 1 apart) or to the humidity chamber (Samples 2 and 2a), and were reweighed at intervals over a period of $21 \mathrm{~d}$. Figure 31 summarizes the weight changes due to moisture uptake by the samples. DRIFT spectra were obtained also over this time period.

DRIFT spectra were obtained using a Nicolet 20SXC Fourier transform infrared (FTIR) spectrometer equipped with a Spectra-Tech 0030-005 "DRIFTS" cell and mercury cadmium telluride (MCT) detector. The sample chamber of the FTIR spectrometer is purged with dry nitrogen boiloff from a liquid nitrogen Dewar. Fifty scans were averaged for each spectrum acquired. The resolution of the instrument was $2 \mathrm{~cm}^{-1}$ (16 K data points).

\section{Results and Discussion}

DRIFT spectra of the "dry" and "wet" samples showed some differences, but these were fairly small and not very reproducible. A background subtraction procedure was used to accentuate the differences in spectra of wet and dry samples. In this procedure, the DRIFT spectrum of a dry sample was subtracted either from that of a wet sample (top trace in Figure 32), or from another dry sample (bottom trace in Figure 32). As Figure 32 shows, there is a fairly noisy peak near $3630 \mathrm{~cm}^{-1}$ in the differential spectrum of the "wet" sample $(9 \%$ water content by weight) that does not appear in the spectrum for the dry sample. The location of this peak in the spectrum agrees reasonably well with transmission IR measurements of moisture in cellulose nitrate (12). This peak was not seen in similar spectra obtained for the same samples two days later, however. 


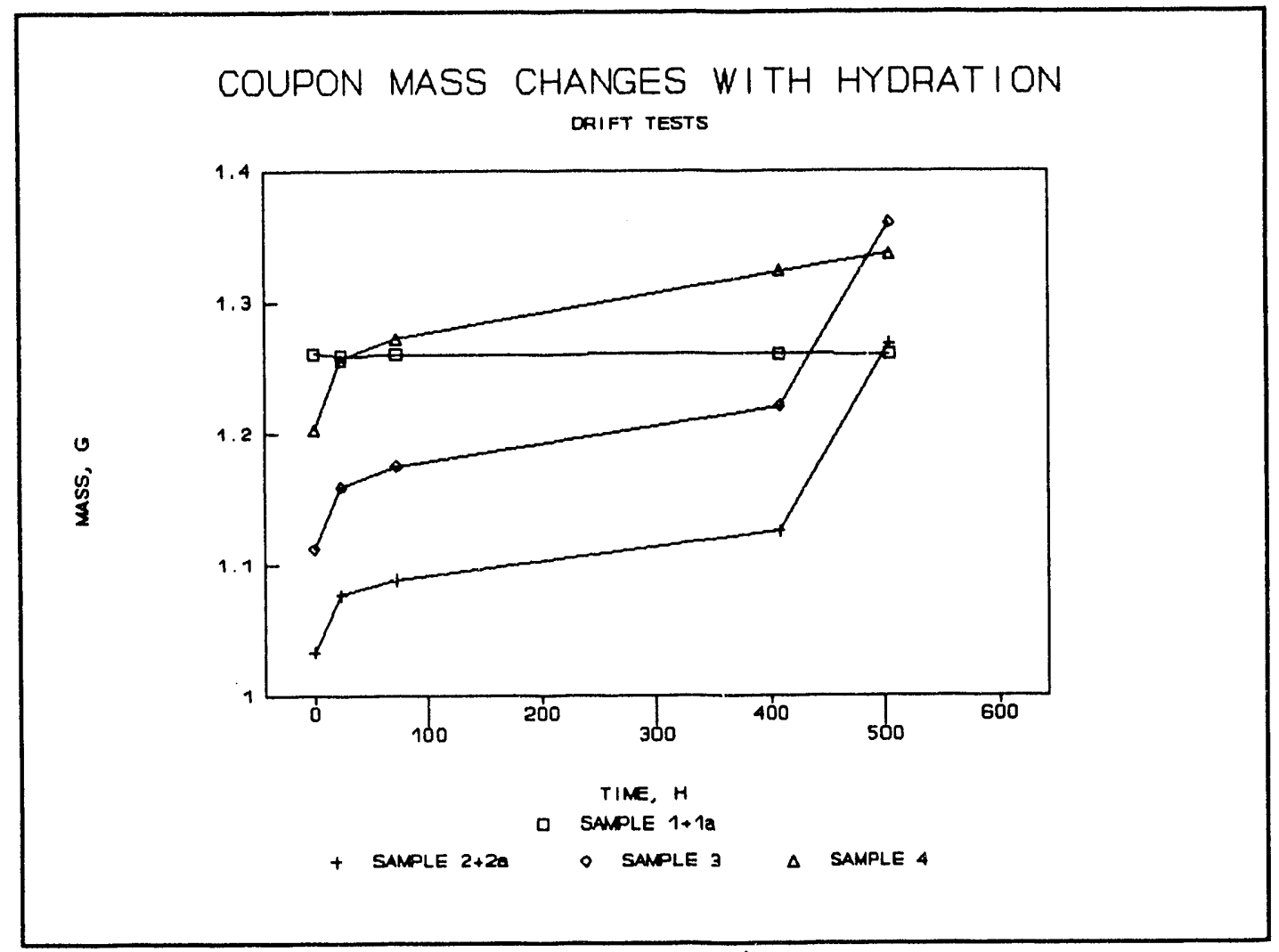

Fig. 31. Mass Changes in CCC Coupons.

At this point, Sample 2 in the humidity chamber was immersed in water for $24 \mathrm{~h}$, and then removed from the water and allowed to equilibrate in the humidity chamber for $24 \mathrm{~h}$. Sample 2 then had a moisture content of 32\%. DRIFT spectra of Sample 2 and Sample 1 (the "dry" sample) were obtained and compared. A large noise content in the DRIFT spectrum of Sample 2 prevented any observation of a contribution from the moisture. 


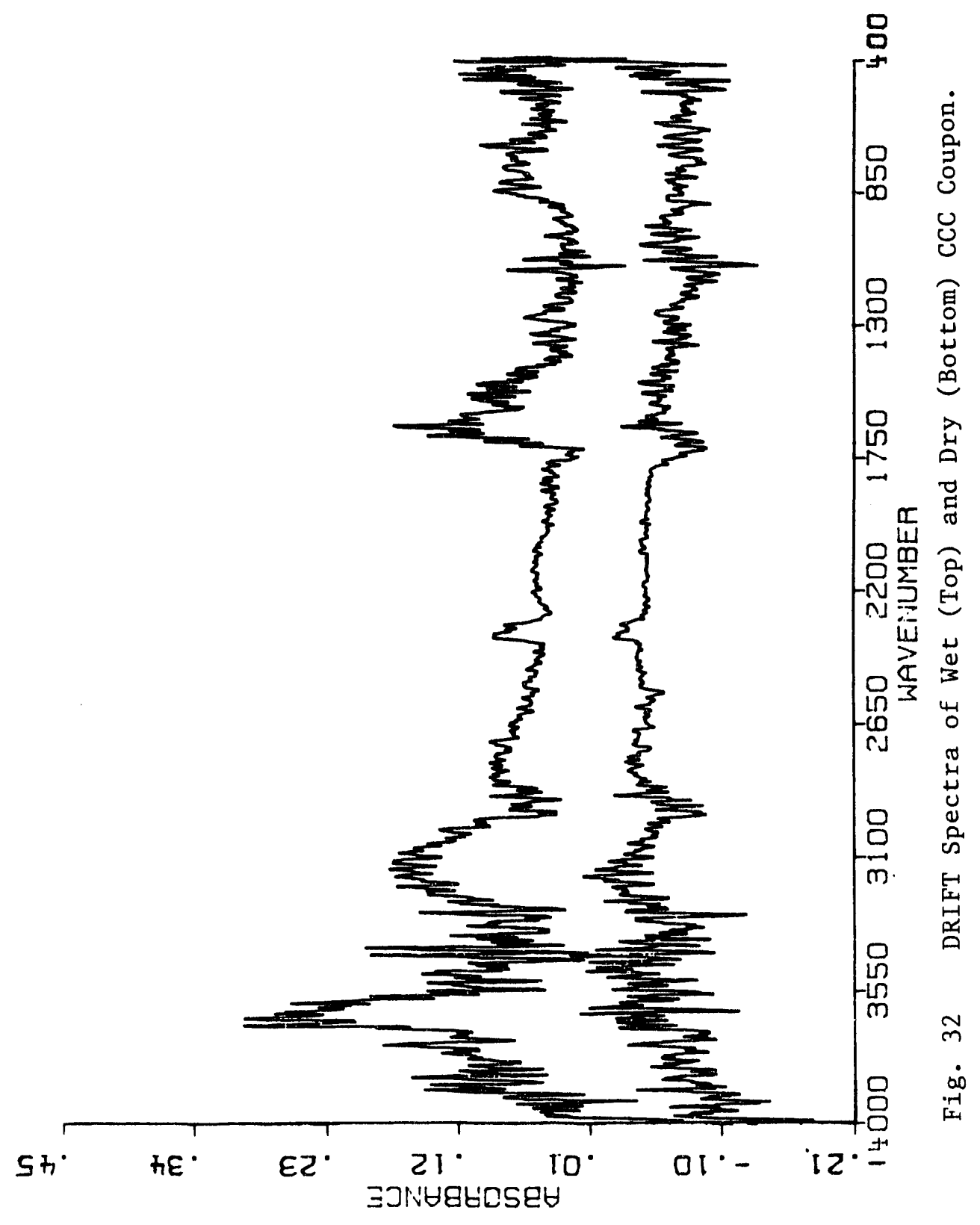


Possible reasons for the lack of reproducibility observed in DRIFT spectra are (a) changes over time in the level of atmospheric moisture in the sample chamber of the spectrometer, and (b) the strong dependence of DRIFT spectra on the light-reflecting properties of the sample surface. Moisture in the sample chamber can be partially compensated for by ensuring equal chamber purge times for wet and dry samples. Problems with sample surface inhomogeneities that adversely affect DRIFT spectra may be eliminated by using other techniques for obtaining infrared spectra. One promising alternative is attenuated total reflectance (ATR), which involves application of modest pressure to bring the sample in contact with a long, thin prism through which the infrared light is passed. Because the sample and prism are pressed together, small irregularities in the sample surface may be smoothed out. Additionally, more of the sample surface will be illuminated by the infrared light in the ATR cell than in the DRIFT cell, yielding a measurement that is averaged over a larger area.

\section{Conclusions and Recommendations}

This preliminary test of one infrared spectroscopy technique for analyzing solid samples was not encouraging in its ability to detect moisture in ccc material. Other infrared surface techniques, such as attenuated total reflectance, should be tested before a final decision is made on the method. 


\section{CONCLUSIONS AND RECOMMENDATIONS}

The performance tests using cellulose test squares spiked with NG suggest that nitroester detection in ccc munitions is possible using indicator strips based upon the modified Griess reagent, and that quantitative information can be derived from the color intensity or optical density. Similarly, the pelletized cupric chloride indicator tests suggest that a ccc moisture indicator is possible, although at this stage, the time to color change appears to be the indicator of moisture concentration. At their present stages of development, the performances suggest that the indicators would be most suitable be for temporary, one-time tests of munitions rather than for a permanent installation on the rounds and continuous indication.

It is recommended that development of the nitroester indicator be continued. Further work on the nitroester indicator must address questions of indicator reagent stability and lifetime, means of potentiating the sensitivity over long periods of time, potential sources of interferences from propellant and case wall degradation, adaptation to munitions walls and current manufacturing techniques, and calibration of response with actual munitions. Similar questions also arise in the further development of indicators for moisture.

The commercial meter for moisture determination in building materials by electrical capacitance measurements is very promising in its potential for an inexpensive, rapid, nondestructive, and portable means of measuring moisture in cce wall. The main work required is to optimize the electrode design(s) for the shapes of munitions, optimization of the measurement frequency, and calibration with munitions. It is strongly recommended that this work be continued, and it appears possible that this device could be brought into use sooner than the indicator strips. 


\section{REFERENCES}

1. C. Y. Manning, "Deterioration of 120-mm Skive Joint in Storage," Technical Report ARAED-TR-87028, U. S. Army Research, Development, and Engineering Center, Picatinny Arsenal, NJ (November, 1987).

2. C. Y. Manning, "Nitrate Ester Migration from Propellant to Combustible Cartridge Case," American Defense Preparedness Association Meeting, Phoenix AZ (March, 1989).

3. J. M. Hoegfeldt, R. E. Isleifson, and G. G. Wittmer, "Adhesives for Joining Combustible Cartridge Case Components, Second and Last Supplementary Report," Engineering Report ER 4561, Honeywell, Minneapolis, MN (October 30, 1984), and previous reports in this series.

4. W. H. Griest, C., K. Bayne, J. E. Caton, R. Baldwin, J. H. Moneyhun, W. M. Caldwell, and H. E. McCoy, "Predictive Modelling and Laboratory Study of Adhesive Joint Deterioration in the M829 APFSDS Kinetic Energy Round," ORNL/TM-12097, Oak Ridge National Laboratory, Oak Ridge, TN (April, 1992).

5. C. -h. Ho, W. H. Griest, and M. R. Guerin, "Survey of Candidate Chemistries for Skive Joint Deterioration Indicator Strip," ORNL/TM-12093, Oak Ridge National Laboratory, Oak Ridge, TN (December 31, 1991).

6. J. M. Hoegfeldt, R. E. Isleifson, and G. G. Wittmer, "Adhesives for Joining Combustible Cartridge Case Components, First Supplementary Report," Engineering Report No. ER 4223, Honeywell, Minneapolis, MN (April 30, 1984).

7. J. W. Pyper et al., "The Measurement of Bound and Free Moisture in Organic Materials by Microwave Methods," Proceedings of the 1985 International Symposium on Moisture and Humidity, Washington D.C. (April 15-18, 1985) p. 909.

8. J. W. Pyper, "The Analysis of Moisture in Solids: A Review of the Last Decade, or From Pande to the Present", UCRL-53447, Lawrence Livermore National Laboratory, Livermore CA (1983).

9. R. L. Ward, J. A. Happe, and J. W. Pyper, "Bound and Free Moisture Studies of Solid Materials by FTNMR." Proceedings of the 1985 International Symposium on Moisture and Humidity, Washington D.C. (April 15-18, 1985) p. 681.

10. M. S. Hodgman, Editor-in-Chief, Handhook of Chemistry and Physics, 41 st Edition, p. 1560, -Chemical Rubber Publishing Company, Cleveland, Ohio. 


\section{REFERENCES}

(continued)

11. W. Meyer and W. Schilz, "Microwave Absorption by Water in Organic Materials." Third International Conference on Dielectric Materials Measurements and Applications, Burmimgham, UK (1973) p. 215.

12. E. P. Kalutskaya, S. S. Gusev, A. V. Kostochko, and T. M. Berdnikova, "Investigation of the Hydration of Cellulose Nitrates using IR Spectroscopy Techniques", Vysokomolekulyarnyye soyedineniya, Ser. A, 23, 1487-1493 (1981). Translated by Language Services, Knoxville, TN. 


\section{APPENDIX}

Instruction Manual for TRAMEX Moisture Encounter Model MF-1

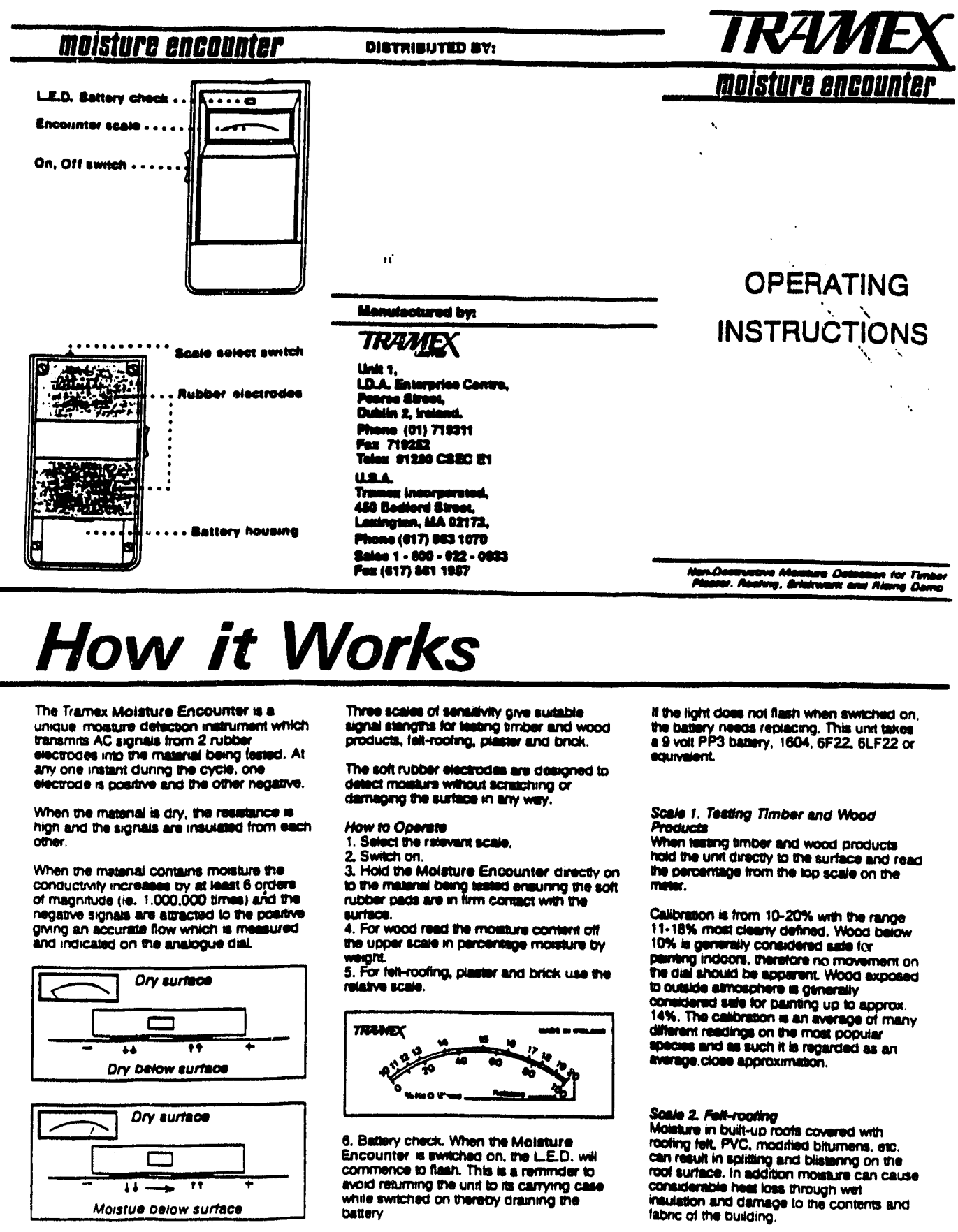


The Molsture Encounter can be used to contirm a new root has been installed dry and to race leaks in an old root.

As there are many difterent types of memorane and insulations it is not possible to calibrate to ove a Dercentage moistur measurement. The Relatwo Scale is used lor checking the difference between wet and dry.

A leak in a bull-up root may enter the bulling a long distance from where the mosture enters the root. Take the Moisture Encounter to the area above the bak. Place thon the root so that the signats penetrate the the root so that the signas ponesult tho lell and indican the signais penceses. By checking acroses the rool. the outine of the "Moisture Prolilo" can be marked with spray pant or chak. By this mothod tho entre wet ared can be cutined thereby leading to the detect which allows water enty

If graver surtacing is present it needs to be removed by scraiching with or similar to ensure ciose contact mith the suriace.

No cutring of the memorane is necessary in order 10 detect motsture. However, It is aways recommended that a core be cut to determine the depth and density of water betore deciding 10 carny out repars.
Scale 3. Plaster, Brickwork

When lesung ptaster, the readings are taken on the relabve scalo. When the reading is in the green area the pleacer is considered sate for the epplicetion of paint. When in the red it is considered wot. Pieses note. The presence of sats on the surtace could give a high reeding.

Masonry wals which have been placed on soil which becomea wet or seturabed or those that have defectrve or non-exstant mosture basmer, often heve moxture mioration into thern. Beceuse of the high cost of remedist work it 4 cential the the extem of the dampres be identiod. Sliding the Moisture Encounter ecroses the well surtece, whth the electronic peds in contact will show the ditierence between contact will show the ditierence besm

Fire damage. Quite ofton a lot of water damage is caused during the lighting of a fire. The Molsture Encounter can be used to check wals. fummure. carpers. drapes elc. and is extremely usetul in the manteniance and renovation of all types of buildings.

\section{Umitations}

On dense concrete mid scale readings may be obtanned even if the materal is ory. When mosture is presem a higher reading will be obtaned 80 a comparson can be made. If a more accurate reeding is

required contect Tramex for dethils of the Tramex Concrese Scanner.
The Mointure Encounter will not delect mousure through certain rubber root membranes due 10 the presence of carbon black liller. These include EPDM's and Buty's. If this is the case. contact Tramex or detads of the Tramex C.R. Scanner which has been specifically developed for nece maberieds.

\section{Guarantes}

Umited guarantes covers repaur required reulting from delects in matenals or workmanship tor a penod of one year from dite of purchase. Proot of purchase is nocesary.

Tramex Limnted accept no responsibility for costs incurred due lo intormavon given by this iratrument

rouing

All Tramex instruments are ngorousty lested b ensure long lite under rougn conditions. Now battenes will need to be occasionally natalled and replacements should be kept in reserve and installed once battery check light (LED) is not constanty lit.

Molsture Encounter. Patem Pending.

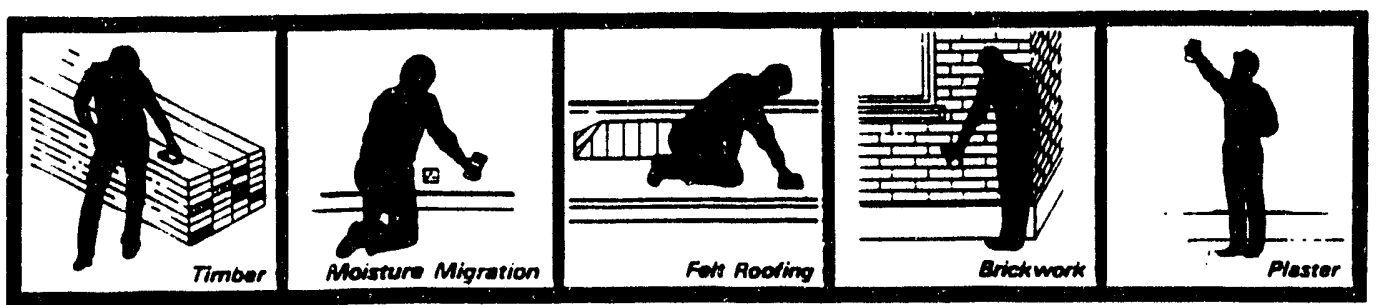




\section{DISTRIBUTION LIST}

No. of Copies

Mr. Paul E. Chiodo

Acting Chief, Nuclear Systems

and Stockpile Reliability Division

ATTN: SMCAR-QAN

Picatinny Arsenal, NJ 07806-5000

Central Research Library

1

Bldg 4500N, MS-6191

Oak Ridge National Laboratory

P.O. Box 2008

Oak Ridge, TN 37831-6286

Document Reference Section

1

Bldg 9711-1, MS-6107

Oak Ridge National Laboratory

P.O. Box 2008

Oak Ridge, TN 37831-6286

Dr. Ronald O. Hultgren

Deputy Assistant Manager

Energy Research and Development

DOE Oak Ridge Field Office

Post Office Box 2008

Oak Ridge, TN 37831-6269

W. H. Griest

5

Bldg 4500S, MS-6120

Oak Ridge National Laboratory

P.O. Box 2008

Oak Ridge, TN 37831-6120

M. R. Guerin

1

Bldg 4500S, MS-6120

Oak Ridge National Laboratory

P.O. Box 2008

Oak Ridge, TN 37831-6120 


\section{DISTRIBUTION LIST}

(continued)

No, of Copies

C.-h. Ho

Bldg 4500S, MS-6120

Oak Ridge National Laboratory

P.O. Box 2008

Oak Ridge, TN 37831-6120

Mr. Robert J. Kuper

Chief, Packaging Division

SMCAR-AEP

Picatinny Arsenal, NJ 07806-5000

Laboratory Records

Bldg 4500S, MS-6285

Oak Ridge National Laboratory

P.O. Box 2008

Oak Ridge, TN 37831-6285

ORNL Patent Office

Bldg 4500N, MS-6258

Oak Ridge National Laboratory

P.O. Box 2008

Oak Ridge, TN 37831-6258

U.S. Department of Energy

Office of Scientific

and Technical Information

P.O. Box 62

?ak Ridge, TN 37831

J. H. Clift

Bldg 9202, MS-8097

Martin Marietta Energy Systems, Inc.

Y-12 Plant

P.O. Box 200 ?

Oak Ridge, TN 37831-8097 


\section{DISTRIBUTION LIST}

(continued)

No. of Copies

R. M. Davis

Bldg 4500N, MS-6195

Oak Ridge National Laboratory

P.O. Box 2008

Oak Ridge, TN 37831-6195

A. M. Ammons

1

Bldg 9209, MS-8096

Martin Marietta Energy Systems

Y-12 Plant

P.O. Box 2009

Oak Ridge, TN 37831-8096

J. H. Hannah

1

Bldg 7601, MS-6304

Oak Ridge National Laboratory

P.O. Box 2008

Oak Ridge, TN 37830-6304

J. H. Moneyhun

Bldg 4500S, MS-6120

Oak Ridge National Laboratory

P.O. Box 2008

Oak Ridge, TN 37831-6120

D. C. Agouridis

Bldg 3500, MS-6010

Oak Ridge National Laboratory

P.O. Box 2008

Oak Ridge, TN 37831-6010

G. B. Hurst

1

Bldg 4500S, MS-6120

Oak Ridge National Laboratory

P.O. Box 2008

Oak Ridge, TN 37831-6120 


\section{DISTRIBUTION LIST}

(continued)

No. of Copies

J. Kevorkian

U.S. Army Armament Research, Development and Engineering Center

ATTN: SMCAR-QAN-P/Mr. Kevorkian

Picatinny Arsenal, NJ 07806-5000

T. M. Gayle

Bldg 3500, MS-6010

Oak Ridge National Laboratory

P.O. Box 2008

Oak Ridge, Tn 37831-6010 

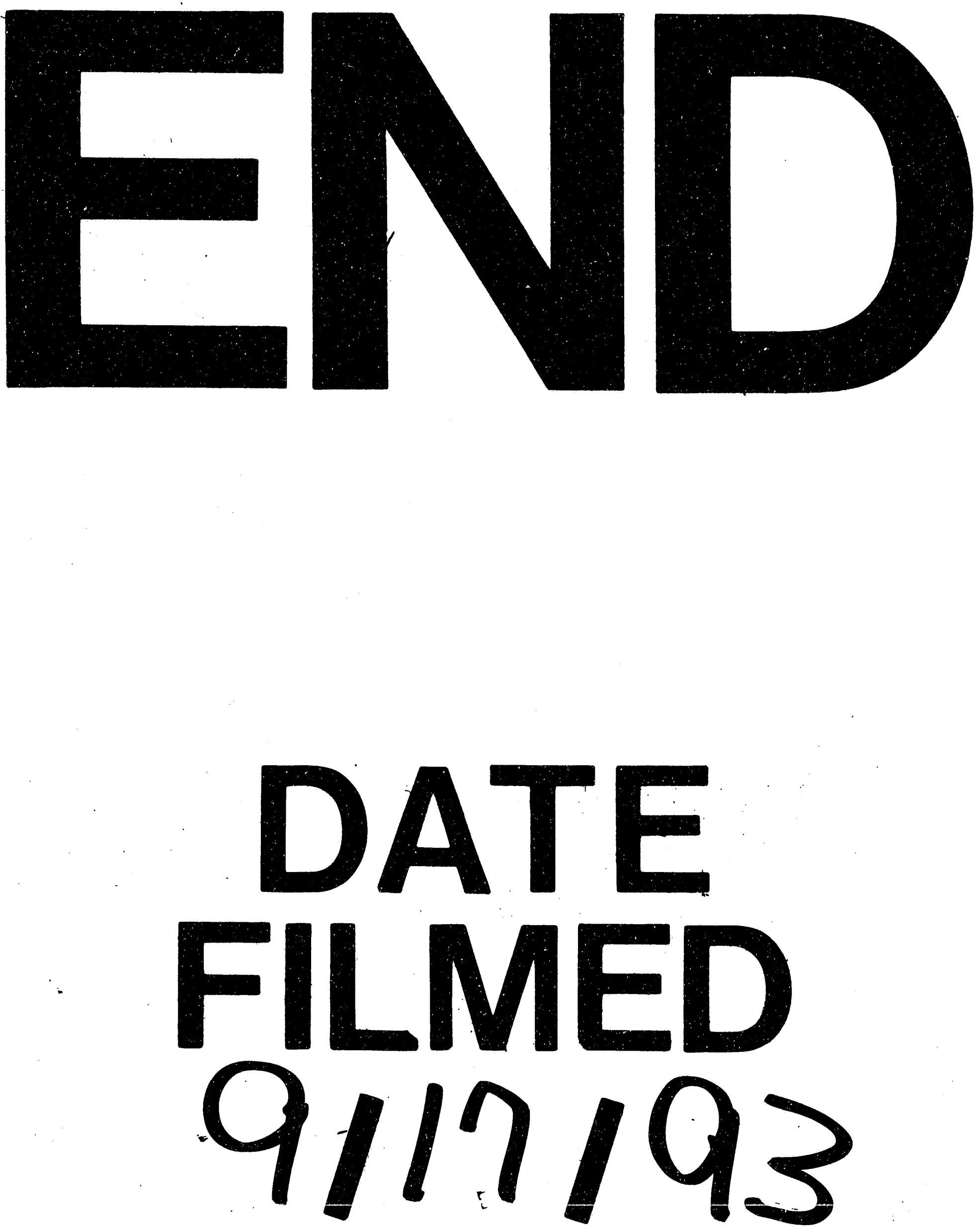
